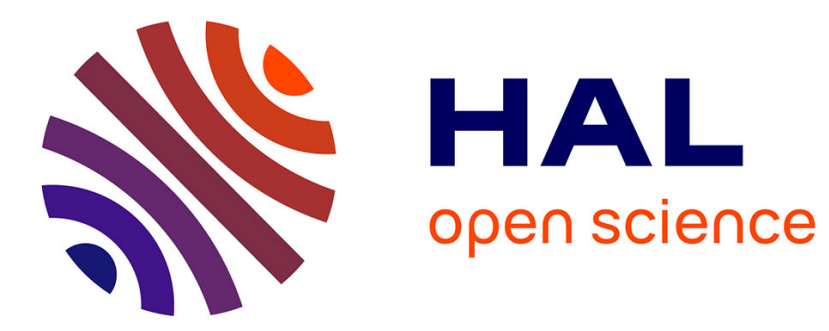

\title{
Decoding the Time-Dependent Response of Bioluminescent Metal-Detecting Whole-Cell Bacterial Sensors
}

\author{
Jérôme F.L. Duval, Christophe Pagnout
}

\section{- To cite this version:}

Jérôme F.L. Duval, Christophe Pagnout. Decoding the Time-Dependent Response of Bioluminescent Metal-Detecting Whole-Cell Bacterial Sensors. ACS Sensors, 2020, 309, pp.article 127751. 10.1021/acssensors.9b00349 . hal-02095865

\section{HAL Id: hal-02095865 \\ https://hal.univ-lorraine.fr/hal-02095865}

Submitted on 30 Apr 2019

HAL is a multi-disciplinary open access archive for the deposit and dissemination of scientific research documents, whether they are published or not. The documents may come from teaching and research institutions in France or abroad, or from public or private research centers.
L'archive ouverte pluridisciplinaire HAL, est destinée au dépôt et à la diffusion de documents scientifiques de niveau recherche, publiés ou non, émanant des établissements d'enseignement et de recherche français ou étrangers, des laboratoires publics ou privés. 


\title{
Decoding the Time-Dependent Response of Bioluminescent Metal-Detecting Whole-Cell Bacterial Sensors
}

\author{
Jérôme F.L. Duval, ${ }^{1, *}$ Christophe Pagnout ${ }^{2}$ \\ ${ }^{1}$ Université de Lorraine, CNRS, LIEC (Laboratoire Interdisciplinaire des Environnements Continentaux), \\ UMR7360, Vandoeuvre-lès-Nancy F-54501, France. \\ ${ }^{2}$ Université de Lorraine, CNRS, LIEC, UMR7360, Campus Bridoux, Metz F-57070, France. \\ ${ }^{*}$ Corresponding author: jerome.duval@univ-lorraine.fr
}

\begin{abstract}
.
The signal produced by aqueous dispersions of bioluminescent, metal-responsive whole-cell bacterial sensors is indicative of the concentration of bioavailable metal ions in solution. The conventional calibration-based strategy followed for measuring this concentration is however inadequate to provide any quantitative prediction of the cells response over time as a function of e.g. their growth features, their defining metal accumulation properties, or the physicochemical medium composition. Such an evaluation is still critically needed for assessing on a mechanistic level the performance of biosensors in terms of metal bioavailability and toxicity monitoring. Herein we report a comprehensive formalism unraveling how the dependence of bioluminescence on time is governed by the dynamics of metal biouptake, by the activation kinetics of lux-based reporter gene, by the ensuing rate of luciferase production, the kinetics of light emission and quenching. It is shown that bioluminescence signal corresponds to the convolution product between two time-dependent functions, one detailing the dynamic interplay of the above microand nanoscale processes, and the other pertaining to the change in concentration of photoactive cell sensors over time. Numerical computations illustrate how the shape and magnitude of the bioluminescence peak(s) are intimately connected to the dependence of the photoactive cells concentration on time and to the magnitudes of Deborah numbers that compare the relevant timescales of the biointerfacial and intracellular events controlling light emission. Explicit analytical expressions are further derived for practical situations where bioluminescence is proportional to the concentration of metal ions in solution. The theory is further quantitatively supported by experiments performed on luminescent cadmium-responsive lux-based Escherichia coli biosensors.
\end{abstract}

Keywords. Whole-cell bacterial sensors, Bioluminescence, Time-dependent response, Dynamics, Metal biouptake, Luciferase, Quenching. 
In a context where anthropogenic activities lead to significant contamination of natural aquatic environments, efficient ecosystem management strategies requires suitable devices to detect trace pollutants such as metals in aqueous solutions. ${ }^{1}$ Conventional physicochemical methods adopted for that purpose (e.g. spectrometry, conductivity, complexometry) often suffer from the complexity of sample preparation protocols, from interference effects associated with the aqueous matrix containing the element to be measured, or the impossibility to assess the bioavailable metal fraction, which is however essential for a proper estimate of toxicity effects on biota. ${ }^{2-6}$ In order to develop reliable methods for a fine monitoring of metals bioavailability and toxicity, the scientific community has resorted to solutions derived from biotechnologies, such as whole-cell microbial biosensors. ${ }^{7-10}$ The latter correspond to genetically modified microorganisms that emit a measurable physical or (electro)chemical signal in the presence of the target metal analyte. ${ }^{7-13}$ Genetic constructions are all based on the same principle and are strongly inspired by bacterial mechanisms of metal homeostasis. ${ }^{7}$ Reporter genes, introduced e.g. in the form of plasmids, are fused to a promoter whose expression is regulated by a regulatory (or repressor) protein that has a strong affinity with the metal element to be detected (Figure 1). In the absence of this element, the expression of reporter genes is repressed by the repressor attached to the promoter. Inactivation of the repressor occurs when the latter forms a complex with the metal ion, thus allowing the expression of reporter genes and the production of so-called reporter proteins (e.g. GFP or luciferase) at the origin of the measured signal (e.g. fluorescence or luminescence). ${ }^{7,8}$ Many so-constructed whole-cell bacterial sensors are listed in the literature ${ }^{12-18}$ with detection limits from a few $\mathrm{nM}$ to ten $\mu \mathrm{M}$.

The response of bacterial metal sensors over time depends on the intracellular concentration of the target metal and, more generically, on the dynamics of the processes that determine the partition of metals at the interphase between the biosensor and the extracellular medium. ${ }^{18}$ Depending on the bacterial systems considered and on medium composition, these processes include the reactive transfer of metals from the solution to the biosurface (e.g. their speciation), ${ }^{3,18-20}$ their passive biosorption, ${ }^{18}$ their internalization and bioaccumulation, ${ }^{21,22}$ or their excretion via efflux pumps. ${ }^{22}$ The biosensor signal is further intimately determined by the production rate of reporter proteins, which necessarily depends on the efficiency with which the transcription of reporter genes is activated by the metal ion-repressor protein complex. ${ }^{18,23-25}$ The physiology of cell sensors is an additional factor controlling their ability to produce light, and any interfering toxic effects generated by the metal species shall necessarily impact their response. These elements illustrate the complexity of the signal emitted by metal-responsive wholecell bacterial sensors, the time dependence and magnitude of which are inherently mediated by interrelated extracellular, biointerfacial and intracellular bio-physicochemical processes. The ensuing lack of predictability of biosensor signals probably explains why this technology has met a limited number of 
commercial achievements for environmental monitoring ${ }^{26,27}$ and why testing in natural aquatic media still remains scarce. ${ }^{12,17}$

Further efforts are thus required to rationalize whole-cell bacterial sensors signals on mechanistic and quantitative levels. The models developed for that purpose mainly rely on systems of differential equations that translate the coupling between kinetics of the successive steps leading to e.g. fluorescence or luminescence emission. ${ }^{18,23,28-31}$ As correctly argued in Ref. [23] where focus is given to bioluminescence, these models generally differ in their degree of sophistication for representing the various molecular determinants that control bioluminescence reaction and catalysis thereof, and thus in their number of introduced parameters, some of them remaining difficult -if not impossible- to measure under realistic sensors exposition conditions. With some exceptions, ${ }^{18}$ most of these approaches have in common that they further discard the complexity of the physicochemical processes governing the bioavailability of the target analyte at the biosensors/solution interface. The impact of cell growth on biosensors response is further sometimes incorrectly formulated by introduction of a simple proportionality factor in theoretical expressions of bioluminescence, ${ }^{24}$ which contrasts with the nonlinearity recently invoked in the noticeable work by Delle Side et al. ${ }^{23}$ In line with the nature of the biosystem they considered, these authors did not account for the possible exchange fluxes of the molecules triggering light production, between cells and external medium. This aspect is however critical for proper assessment of the luminescence emitted by metal-responsive whole-cell biosensors. ${ }^{18}$

In view of the above elements, we report herein a conceptual framework allowing a full integration of the dynamic coupling between (i) the processes that regulate the partitioning of metals at the interphase between aqueous medium and luminescent metal-responsive whole-cell bacterial sensors, (ii) the formation of metal-repressor protein complex, which initiates reporter gene expression, (iii) the production of luciferase and the ensuing light emission and quenching, and finally (iv) the processes leading to time variations in concentration of photoactive cells. Illustrative numerical computations, supported by tractable analytical developments, decipher the impact of each of the above process on the bioluminescence response. Implications for bioluminescence data interpretation are further discussed, and analysis of the bioluminescence response of cadmium-detecting lux-based Escherichia coli biosensors supports theory.

\section{THEORY}

Setting the stage. In the following developments, we consider a dispersion of genetically-engineered (lux operon-based $^{7,32}$ ) bacteria that produce light after their accumulation of metal ions ( $\mathrm{M}$ for short) present in solution at bulk concentration denoted as $C_{\mathrm{M}}^{*}\left(\mathrm{~mol} \mathrm{~m}^{-3}\right)$. Depending on solution composition and on the amount of available nutrients therein, the number $N$ of photoactive metal-responsive bacteria may vary 
with time $t$ due to cell growth, to inactivation of some of the biosensors or to metal-induced toxicity effects. Accordingly, we define the time-dependent concentration $c_{\mathrm{p}}(t)\left(\mathrm{m}^{-3}\right)$ of photoactive cells by $c_{\mathrm{p}}(t)=N(t) / V_{\mathrm{T}}$ with $V_{\mathrm{T}}$ the volume of the dispersing medium, and $t=0$ the initial time referring to the introduction of $\mathrm{M}$ in the biosensors dispersion (Figure 1A). For the sake of demonstration, we consider practical situations where metal speciation in solution is not significant ${ }^{18,33}$ and metal depletion from bulk solution as a result of metal bioaccumulation is absent, which ensures that $c_{\mathrm{M}}^{*}$ does not depend on time. ${ }^{34}$ In line with standard experimental conditions, the cell dispersion is sufficiently dilute to ignore possible overlap of metal diffusion layers and electric double layers developed around the (negatively charged $^{35}$ ) cells. ${ }^{18,22,34}$ The bioluminescence produced at $t$ by the metal-sensing whole-cell bacterial sensors is hereafter denoted as $\mathcal{L} \mathrm{um}(t)$ (expressed in counts $\mathrm{s}^{-1}$ or photons $\mathrm{s}^{-1}$ ). The cascade of events leading to photons emission by such biosystems includes (Figures 1B,C): (a) the conductive diffusion of $\mathrm{M}$ from bulk solution to the charged bacterial surface, followed by $M$ internalization and possible excretion via efflux pumps, ${ }^{18,22,34,36,37}$ (b) $\mathrm{M}$ binding to the regulatory (repressor) proteins denoted as $\mathrm{P}_{\text {reg }}$ (e.g. ZntR), ${ }^{7,18,38,39}$ (c) interaction of the formed metal-repressor protein complex, $\mathrm{MP}_{\text {reg, with a promoter }}$ placed upstream the (lux-based) reporter gene, which in turn initiates gene transcription and leads to the production of the reporter protein luciferase that plays a central role in catalyzed bioluminescent reaction, ${ }^{7,32}$ (d) the subsequent emission of photons $(h v=490 \mathrm{~nm})$ and light quenching. ${ }^{23}$ The two following sections detail the theoretical formulations of processes (a)-(b) and (c)-(d), respectively. A glossary of symbols is given in Supporting Information.

Partitioning dynamics of metal ions at the cell sensor/solution interface. The concentration of bioavailable $\mathrm{M}$, hereafter denoted as $c_{\mathrm{M}, \mathrm{f}}^{*}$, refers to the fraction of metal ions that can be uptaken by the cell sensors. In the absence of significant metal speciation in solution, $c_{\mathrm{M}, \mathrm{f}}^{*}$ may differ from $C_{\mathrm{M}}^{*}$ due to rapid adsorption of $\mathrm{M}$ at (passive) cell surface sites. ${ }^{18}$ The latter process does not lead to metal biouptake, unlike the adsorption of $\mathrm{M}$ at dedicated metal transporter sites. ${ }^{40}$ In the practical limit of linear Henry passive biosorption of $\mathrm{M}, c_{\mathrm{M}, \mathrm{f}}^{*}$ is related to $c_{\mathrm{M}}^{*} v^{18}$

$$
c_{\mathrm{M}, \mathrm{f}}^{*}(t) / c_{\mathrm{M}}^{*}=1-\beta_{\mathrm{a}} S_{\mathrm{a}} \hat{K}_{\mathrm{H}} c_{\mathrm{p}}(t)
$$

, where $S_{\mathrm{a}}=4 \pi a^{2}$ is the surface area of an individual biosensor assimilated to a sphere of radius $a$. $\hat{K}_{\mathrm{H}}$ (m) in eq 1 is the Henry coefficient associated to $\mathrm{M}$ passive biosorption, and $\beta_{\mathrm{a}}$ corresponds to the dimensionless Boltzmann accumulation factor of $M$ at the charged cell envelope. This factor depends on the electrostatic cell surface properties, on solution ionic strength and $\mathrm{pH}^{18}$ and it can be evaluated from 
cell electrophoretic mobility measurements and proper interpretation thereof. ${ }^{18,35,41}$ Metal biouptake proceeds here according to Michaelis-Menten mechanism, ${ }^{42}$ which corresponds to a fast langmuirian adsorption of $\mathrm{M}$ at the active transporter sites located at the membrane surface, followed by a first-order rate-limiting internalisation step with kinetic constant $k_{\text {int }} \cdot{ }^{18,22,34,36,40,43}$ The corresponding biouptake flux $J_{\mathrm{u}}\left(\mathrm{mol} \mathrm{m} \mathrm{m}^{-2} \mathrm{~s}^{-1}\right)$ is then defined by $J_{\mathrm{u}}(t) / J_{\mathrm{u}}^{*}=c_{\mathrm{M}}^{a}(t) /\left(K_{\mathrm{M}}+c_{\mathrm{M}}^{a}(t)\right)$ where $J_{\mathrm{u}}^{*}=K_{\mathrm{H}} k_{\mathrm{int}} K_{\mathrm{M}}$ is the maximum achievable $\mathrm{M}$ uptake flux $\left(\mathrm{mol} \mathrm{m}^{-2} \mathrm{~s}^{-1}\right), K_{\mathrm{M}}\left(\mathrm{mol} \mathrm{m}^{-3}\right)$ the reciprocal of the $\mathrm{M}$ affinity for the transporter sites, $K_{\mathrm{H}}(\mathrm{m})$ the corresponding Henry adsorption coefficient, and $c_{\mathrm{M}}^{a}(t)$ is the $\mathrm{M}$ concentration at the membrane surface at $t$ (Figure 1C). Under steady-state metal transport condition achieved within ms timescale for microorganisms, ${ }^{18,22,34,36,37}$ the flux $J_{\mathrm{u}}(t)$ satisfies at any $t^{22,34}$

$$
J_{\mathrm{u}}(t)=J_{\text {diff }}(t)+k_{\mathrm{e}} \phi_{\mathrm{M}}(t)
$$

, where $J_{\text {diff }}\left(\mathrm{mol} \mathrm{m}^{-2} \mathrm{~s}^{-1}\right)$ is the metal diffusion flux from solution to the membrane surface, and $k_{\mathrm{e}} \phi_{\mathrm{M}}$ represents the excretion flux of intracellular $\mathrm{M}^{40}$ with $k_{\mathrm{e}}\left(\mathrm{s}^{-1}\right)$ the excretion kinetic constant and $\phi_{\mathrm{M}}(\mathrm{mol}$ $\mathrm{m}^{-2}$ ) the intracellular concentration of $\mathrm{M}$ expressed per unit cell surface area (Figure 1C). Solving for the steady-state Nernst-Planck equation, the flux $J_{\text {diff }}$ is written $J_{\text {diff }}(t)=\bar{f}_{\mathrm{el}} D_{\mathrm{M}}\left(c_{\mathrm{M}, \mathrm{f}}^{*}(t)-\beta_{\mathrm{a}}^{-1} c_{\mathrm{M}}^{a}(t)\right) / a$ ,$^{22,34}$ with $D_{\mathrm{M}}$ the diffusion coefficient of $\mathrm{M}$ in solution. The dimensionless factor $\bar{f}_{\mathrm{el}}$ corrects the rate of $\mathrm{M}$ diffusion for interfacial cell electrostatics and for the presence of any peripheral soft bio-layer that potentially acts as a barrier against metal diffusion. ${ }^{22,34,44}$ For microorganisms devoid of such a layer, we have $\bar{f}_{\mathrm{el}} \sim 1$ under solution ionic strength conditions of practical interest (typically $1 \mathrm{mM}$ to $100 \mathrm{mM}$ ). ${ }^{44}$ Substituting the expressions of $J_{\text {diff }}$ and $J_{\mathrm{u}}$ into eq 2, it is shown that $J_{\mathrm{u}}$ is provided by eq 2 with $J_{\text {diff }}(t)$ defined by (details in Supporting Information, SI-1)

$$
\frac{J_{\mathrm{diff}}(t)}{J_{\mathrm{u}}^{*}}=\frac{\bar{c}_{\mathrm{M}, \mathrm{f}}^{*}(t)}{2 B n^{-1}}\left[1+\frac{1+B n^{-1}\left(1-\frac{k_{\mathrm{e}} \phi_{\mathrm{M}}(t)}{J_{\mathrm{u}}^{*}}\right)}{\bar{c}_{\mathrm{M}, \mathrm{f}}^{*}(t)}\right]\left\{1-\left[1-\frac{4 B n^{-1}\left[1-\frac{k_{\mathrm{e}} \phi_{\mathrm{M}}(t)}{J_{\mathrm{u}}^{*}}\left(1+\frac{1}{\bar{c}_{\mathrm{M}, \mathrm{f}}^{*}(t)}\right)\right]}{\bar{c}_{\mathrm{M}, \mathrm{f}}^{*}(t)\left[1+\frac{1+B n^{-1}\left(1-\frac{k_{\mathrm{e}} \phi_{\mathrm{M}}(t)}{J_{\mathrm{u}}^{*}}\right)}{\bar{c}_{\mathrm{M}, \mathrm{f}}^{*}(t)}\right]}\right]^{2}\right] .
$$


Equation 3 involves the dimensionless concentration $\bar{c}_{\mathrm{M}, \mathrm{f}}^{*}=\beta_{a} c_{\mathrm{M}, \mathrm{f}}^{*} / K_{\mathrm{M}}$ of bioavailable $\mathrm{M}$ in solution that is related to the dimensionless total $\mathrm{M}$ concentration $\bar{c}_{\mathrm{M}}^{*}=\beta_{a} c_{\mathrm{M}}^{*} / K_{\mathrm{M}} \quad$ via $\bar{c}_{\mathrm{M}, \mathrm{f}}^{*}(\bar{t}) / \bar{c}_{\mathrm{M}}^{*}=1-\varepsilon_{\mathrm{ads}} \bar{c}_{\mathrm{p}}(\bar{t})$ (see eq 1 ) where $\varepsilon_{\mathrm{ads}}=\beta_{\mathrm{a}} S_{\mathrm{a}} \hat{K}_{\mathrm{H}} c_{\mathrm{p}}^{\max }$ is the dimensionless scalar quantifying the passive metal biosorption process in the linear Henry regime, and $\bar{c}_{\mathrm{p}}(\bar{t})=c_{\mathrm{p}}(\bar{t}) / c_{\mathrm{p}}^{\max }$ is the normalized concentration of photoactive cells with $c_{\mathrm{p}}^{\max }$ the maximum photoactive cells concentration in the investigated time range. $B n$ in eq 3 corresponds to the dimensionless bioavailability number $B n=\left(D_{\mathrm{M} \text {,out }} \bar{f}_{\mathrm{el}} a^{-1}\right) /\left(k_{\mathrm{int}} K_{\mathrm{H}} \beta_{\mathrm{a}}\right)$ corrected for cell surface electrostatics. ${ }^{22,34}$ The extremes $1 / B n<<1$ and $1 / B n>>1$ correspond to situations where the rate of metal biouptake is determined by the kinetics of $\mathrm{M}$ internalization (as assumed within the conventional Biotic Ligand Model ${ }^{45}$ ) and by the diffusive transport of $M$ to the biosurface, respectively. ${ }^{22,34}$ In the absence of $M$ excretion and for insignificant passive biosorption of $\mathrm{M}\left(c_{\mathrm{M}, \mathrm{f}}^{*}(t) \approx c_{\mathrm{M}}^{*}\right)$, the dimensionless flux $\bar{J}_{\mathrm{u}}=J_{\mathrm{u}} / J_{\mathrm{u}}^{*}$ identifies with $J_{\mathrm{diff}} / J_{\mathrm{u}}^{*}$ and becomes independent of time. It first increases linearly with $\bar{c}_{\mathrm{M}}^{*}$ (slope $1 /\left(1+B n^{-1}\right)$, details in SI-1), then gradually levels off until it reaches unity at $\bar{c}_{M}^{*}>>1$, which corresponds to saturation of all metal transporter sites.

Assuming that regulatory proteins $\mathrm{P}_{\text {reg }}$ exhibit the strongest metal binding capacity among all other intracellular $\mathrm{M}$ chelators, as verified for other metalloproteins, ${ }^{37}$ the concentration $\phi_{\mathrm{M}}(t)$ of internalized $M$ verifies (Figures 1B,C)

$$
\frac{\mathrm{d}\left[\phi_{\mathrm{M}}(t) c_{\mathrm{p}}(t)\right]}{\mathrm{d} t}=\left[J_{\mathrm{u}}(t)-k_{\mathrm{e}} \phi_{\mathrm{M}}(t)\right] c_{\mathrm{p}}(t)-k_{\mathrm{a}} \rho_{\mathrm{s}}^{V_{\mathrm{i}}} \phi_{\mathrm{M}}(t) c_{\mathrm{p}}(t)+k_{\mathrm{d}} \phi_{\mathrm{c}}(t) c_{\mathrm{p}}(t)
$$

, where $J_{\mathrm{u}}(t)$ is defined by eqs $2-3, k_{\mathrm{a}}\left(\mathrm{mol}^{-1} \mathrm{~m}^{3} \mathrm{~s}^{-1}\right)$ and $k_{\mathrm{d}}\left(\mathrm{s}^{-1}\right)$ are the kinetic constants for the formation and dissociation of the metal-regulatory protein complexes $\mathrm{MP}_{\text {reg }}$, respectively. $\rho_{\mathrm{S}}^{V_{\mathrm{i}}}\left(\mathrm{mol} \mathrm{m}^{-3}\right)$ in eq 4 pertains to the concentration of metal-binding sites carried by the proteins $\mathrm{P}_{\text {reg }}$ and smeared-out over the volume $V_{\mathrm{i}}=4 \pi a^{3} / 3$, while $\phi_{\mathrm{c}}(t)\left(\mathrm{mol} \mathrm{m}^{-2}\right)$ represents the intracellular concentration of $\mathrm{MP}_{\text {reg }}$ complexes expressed per unit cell surface area. The counterpart of eq 4 for chemodynamics of $\mathrm{MP}_{\text {reg }}$ complexes reads as

$$
\frac{\mathrm{d}\left[\phi_{\mathrm{c}}(t) c_{\mathrm{p}}(t)\right]}{\mathrm{d} t}=k_{\mathrm{a}} \rho_{\mathrm{s}}^{V_{\mathrm{i}}} \phi_{\mathrm{M}}(t) c_{\mathrm{p}}(t)-k_{\mathrm{d}} \phi_{\mathrm{c}}(t) c_{\mathrm{p}}(t)
$$


Kinetics of luciferase production and expression of ensuing bioluminescence. Luciferase production sets in after activation of the reporter gene that follows the interaction between promoter and metalregulatory protein complexes (Figures 1B,C). The corresponding luciferase production flux generated by the assembly of biosensors in the dispersion volume $V_{\mathrm{T}}$ may be formulated according to

$$
S_{\mathrm{a}} \frac{\mathrm{d}\left[\phi_{\mathrm{Luc}}(t) c_{\mathrm{p}}(t)\right]}{\mathrm{d} t}=k_{\mathrm{f}} \frac{\left[\phi_{\mathrm{c}}(t) S_{\mathrm{a}} c_{\mathrm{p}}(t)\right]^{n}}{K_{\mathrm{Hi}}{ }^{n}+\left[\phi_{\mathrm{c}}(t) S_{\mathrm{a}} c_{\mathrm{p}}(t)\right]^{n}}-k_{\mathrm{r}} S_{\mathrm{a}} \phi_{\mathrm{Luc}}(t) c_{\mathrm{p}}(t)
$$

, where $\phi_{\mathrm{Luc}}(t)\left(\mathrm{mol} \mathrm{m}^{-2}\right)$ is the intracellular concentration of luciferase at $t$ per unit cell surface area, $k_{\mathrm{f}}$ $\left(\mathrm{mol} \mathrm{m} \mathrm{s}^{-3}\right)$ and $k_{\mathrm{r}}\left(\mathrm{s}^{-1}\right)$ the kinetic constants for luciferase production and degradation, respectively. The half-life $1 / k_{\mathrm{r}}$ of the luciferase enzyme reflects the activity performance of luciferase in the bioluminescence reaction. The rate of change in luciferase concentration over time is here described by the Hill function $\frac{\left[\phi_{\mathrm{c}}(t) S_{\mathrm{a}} c_{\mathrm{p}}(t)\right]^{n}}{K_{\mathrm{Hi}}{ }^{n}+\left[\phi_{\mathrm{c}}(t) S_{\mathrm{a}} c_{\mathrm{p}}(t)\right]^{n}},{ }^{25}$ classically used to model regulation of gene expression by chemicals (here the $\mathrm{MP}_{\text {reg }}$ complexes). In this function, the Hill constant $K_{\mathrm{Hi}}\left(\mathrm{mol} \mathrm{m}^{-3}\right)$ relates to the dissociation constant between promoter and $\mathrm{MP}_{\text {reg }}$ complex and may be viewed as the concentration of $\mathrm{MP}_{\text {reg }}$ at which half of the reporter genes are 'switched on'. The Hill cooperative binding coefficient $n(>0)$ reflects the possible increase $(n>1)$ or decrease $(n<1)$ of the binding affinity of $\mathrm{MP}_{\text {reg }}$ for the promoter as the concentration of available ligands is reduced. ${ }^{25}$

The number of luciferase molecules produced in the volume $V_{\mathrm{T}}$ within an infinitesimal time delay $\mathrm{d} \tau$ is $S_{\mathrm{a}} V_{\mathrm{T}} \frac{\mathrm{d}\left[\phi_{\mathrm{Luc}}(\tau) c_{\mathrm{p}}(\tau)\right]}{\mathrm{d} \tau} \mathrm{d} \tau$. Following the original idea by Delle Side et al., ${ }^{23}$ the existence of luminescence quenching (i.e. a loss of signal over time ${ }^{18,23}$ ) implies that the number of photons-emitting luciferase molecules at $t$ is generally lower than that at $t-\mathrm{d} \tau$. Denoting as $q(t, \tau)$ the function that quantifies the decrease in the amount of photoactive luciferase at $t$ as compared to that at $\tau(<t)$, the luminescence generated by the ensemble of metal-responsive whole-cell sensors at $t$ is provided by ${ }^{23}$

$$
\mathcal{L} \operatorname{Lum}(t)=k_{v} S_{\mathrm{a}} V_{\mathrm{T}} \int_{0}^{t} q(t, \tau) \frac{\mathrm{d}\left[\phi_{\mathrm{Luc}}(\tau) c_{\mathrm{p}}(\tau)\right]}{\mathrm{d} \tau} \mathrm{d} \tau
$$

, where $k_{v}$ (counts $\mathrm{s}^{-1} \mathrm{~mol}^{-1}$ ) is the kinetic constant for photons production per mole of luciferase molecules. To further proceed, we adopt a tractable rectangular expression for $q(t, \tau)^{23}$ written here in 
terms of the Heaviside function $H(t, \tau)=\left\{\begin{array}{l}0 \text { for } t-\tau \leq 0 \\ 1 \text { for } t-\tau>0\end{array}\right.$ according to $q(t, \tau)=H(t, \tau)-H\left(t-\tau, \tau_{\mathrm{q}}\right)$ with $\tau_{\mathrm{q}}$ the characteristic quenching timescale. This form of $q$ states that the luciferase molecules produced at time $\tau$ remain photoactive within the time interval $\left[\tau ; \tau+\tau_{\mathrm{q}}\right]$ and their photoactivity drops to 0 for $t>\tau+\tau_{\mathrm{q}} \cdot{ }^{23}$ In turn, eq 7 becomes

$$
\mathcal{L} \operatorname{Lum}(t)=k_{v} S_{\mathrm{a}} V_{\mathrm{T}}\left[\phi_{\mathrm{Luc}}(t) c_{\mathrm{p}}(t)-H\left(t, \tau_{\mathrm{q}}\right) \phi_{\mathrm{Luc}}\left(t-\tau_{\mathrm{q}}\right) c_{\mathrm{p}}\left(t-\tau_{\mathrm{q}}\right)\right]
$$

, where the condition $\operatorname{Lum}(t=0)=0$ is adopted, consistent with our choice of boundaries $\phi_{\mathrm{M}}(0)=\phi_{\mathrm{c}}(0)=\phi_{\mathrm{Luc}}(0)=0$. The set of coupled eqs 4-6 and 8 fully defines the time-dependent concentrations $\phi_{\mathrm{M}}(t), \phi_{\mathrm{c}}(t), \phi_{\mathrm{Luc}}(t)$ and the corresponding bioluminescence $\mathcal{L} \mathrm{um}(t)$. After some algebra, eqs 4-6 and 8 may be written in the convenient dimensionless forms detailed in SI-1 (eqs S4-S7) after introduction of the following key dimensionless quantities: (i) the dimensionless time $\bar{t}=t / \tau_{\mathrm{a}}$ with $\tau_{\mathrm{a}}=1 /\left(k_{\mathrm{a}} \rho_{\mathrm{s}}^{V_{\mathrm{i}}}\right)$ the characteristic timescale for association of intracellular $\mathrm{M}$ with the regulatory protein $\mathrm{P}_{\text {reg, }}$ (ii) the dimensionless concentration of intracellular $\mathrm{M}$ defined by $\Phi_{\mathrm{M}}(t)=\left(J_{\mathrm{u}}^{*} \tau_{\mathrm{a}}\right)^{-1} \phi_{\mathrm{M}}(t) \bar{c}_{\mathrm{p}}(t)$, (iii) the dimensionless concentration of $\mathrm{MP}_{\text {reg }}$ complex given by $\Phi_{\mathrm{c}}(t)=\left(J_{\mathrm{u}}^{*} \tau_{\mathrm{d}}\right)^{-1} \phi_{\mathrm{c}}(t) \bar{c}_{\mathrm{p}}(t)$ with $\tau_{\mathrm{d}}=1 / k_{\mathrm{d}}$ the dissociation timescale of a complex, (iv) the dimensionless stability constant $\bar{K}=\tau_{\mathrm{d}} / \tau_{\mathrm{a}}$ of $\mathrm{MP}_{\text {reg }}$ complex, and (v) the dimensionless concentration of luciferase, $\Phi_{\mathrm{Luc}}(t)=\left(\frac{S_{\mathrm{a}} c_{\mathrm{p}}^{\max } \bar{K}_{\mathrm{Hi}}{ }^{n}}{k_{\mathrm{f}} \tau_{\mathrm{a}}}\right) \phi_{\mathrm{Luc}}(t) \bar{c}_{\mathrm{p}}(t)$, which involves the dimensionless Hill stability constant $\bar{K}_{\mathrm{Hi}}$ defined by $\bar{K}_{\mathrm{Hi}}=K_{\mathrm{Hi}} /\left(J_{\mathrm{u}}^{*} \tau_{\mathrm{a}} \bar{K} S_{\mathrm{a}} c_{\mathrm{p}}^{\max }\right)$. Unless otherwise stated, for given time-dependent concentration $\bar{c}_{\mathrm{p}}(\bar{t})$ of photoactive cells, for prescribed features of M biouptake and passive biosorption (i.e. at fixed $B n^{-1}, K_{\mathrm{M}}, J_{\mathrm{u}}^{*}$ and $\varepsilon_{\text {ads }}$ ), for given $n, \bar{K}, \bar{K}_{\mathrm{Hi}}$ and fixed values of the Deborah numbers $k_{\mathrm{e}} \tau_{\mathrm{a}}, k_{\mathrm{r}} \tau_{\mathrm{a}}$ and $\tau_{\mathrm{q}} / \tau_{\mathrm{a}}$, the dimensionless forms of eqs 4-6 and 8 were solved in Mathcad 15 calculus environment using an hybrid solver based on either Adams or backward differentiation methods depending on the stiffness of the set of differential equations. ${ }^{46,47}$ Convergency of the numerical results was systematically verified by comparison with predictions from the analytical developments elaborated in the next section under metal concentration conditions of practical interest. 


\section{Explicit analytical expressions for the bioluminescence.}

In the linear Henry metal biouptake regime, the bioavailable metal concentration in solution is so low that the condition $\bar{c}_{\mathrm{M}, \mathrm{f}}^{*}<<1$ is verified. In this regime, and further assuming that passive biosorption of $\mathrm{M}$ is not significant (i.e. $\varepsilon_{\text {ads }} \rightarrow 0$ ), the time-dependent bioluminescence $\mathcal{L} \operatorname{um}(\bar{t})$ is defined by the combination of eqs S7, S10 and S14 derived in SI-2. The linear Hill regime corresponds to metal concentrations sufficiently low for the production rate of luciferase to increase linearly with $\Phi_{\mathrm{c}}$. This latter regime is achieved for bio-systems defined by the absence of Hill binding cooperativity (i.e. $n=1$ )$^{23,48}$ and pending the condition $\Phi_{\mathrm{c}}(\bar{t}) / \bar{K}_{\mathrm{Hi}}<<1$ is satisfied. For metal concentrations in line with both linear Henry metal biouptake and linear Hill regimes, the expression of the bioluminescence emitted by the assembly of $N$ biosensors in the limit $\varepsilon_{\text {ads }} \rightarrow 0$ reduces to (details in SI-3)

$$
\mathcal{L} u m(\bar{t})=\Omega\left\{g(\bar{t}) \otimes \bar{c}_{\mathrm{p}}(\bar{t})-H\left(\bar{t}, \frac{\tau_{\mathrm{q}}}{\tau_{\mathrm{a}}}\right) \int_{0}^{\bar{t}-\frac{\tau_{\mathrm{q}}}{\tau_{\mathrm{a}}}} g\left(\bar{t}-\frac{\tau_{\mathrm{q}}}{\tau_{\mathrm{a}}}-\xi\right) \bar{c}_{\mathrm{p}}(\xi) \mathrm{d} \xi\right\}
$$

, where $\Omega=k_{v} V_{\mathrm{T}} k_{\mathrm{f}} \tau_{\mathrm{a}} / \bar{K}_{\mathrm{Hi}}\left(\right.$ counts s${ }^{-1}$ ), the symbol $\otimes$ is the convolution product operator, and $g$ is the time-dependent dimensionless function defined by

$$
\begin{array}{r}
g(\bar{t})=\frac{\bar{J}_{\mathrm{u}, 0}}{2 \Lambda\left(1-\frac{x}{k_{\mathrm{r}} \tau_{\mathrm{a}}}+\bar{K}\left(1+x-k_{\mathrm{r}} \tau_{\mathrm{a}}\right)\right.}\left\{\left(1-\frac{2 x}{k_{\mathrm{r}} \tau_{\mathrm{a}}[1+\bar{K}(1+x+2 \Lambda)]}\right)\left(\mathrm{e}^{-\left(\Lambda+\frac{1+\bar{K}(1+x)}{2 \bar{K}}\right) \bar{t}}-1\right)\right. \\
-\left(1-\frac{2 x}{k_{\mathrm{r}} \tau_{\mathrm{a}}[1+\bar{K}(1+x-2 \Lambda)]}\right)\left(\mathrm{e}^{\left.\left(\mathrm{C}^{\left(\Lambda-\frac{1+\bar{K}(1+x)}{2 \bar{K}}\right) \bar{t}}-1\right)-\frac{2 \Lambda}{k_{\mathrm{r}} \tau_{\mathrm{a}}}\left(\mathrm{e}^{-k_{\mathrm{r}} \tau_{\mathrm{a}} \bar{t}}-1\right)\right\}}\right.
\end{array}
$$

, with $x=k_{\mathrm{e}} \tau_{\mathrm{a}} /\left(1+B n^{-1}\right), \Lambda=\frac{1+(1+x) \bar{K}}{2 \bar{K}} \sqrt{1-\frac{4 \bar{K} x}{[1+(1+x) \bar{K}]^{2}}}$ and $\bar{J}_{\mathrm{u}, 0}$ the dimensionless metal bioaccumulation flux $\bar{J}_{\mathrm{u}, 0}=\bar{c}_{\mathrm{M}}^{*} /\left(1+B n^{-1}\right)$. The latter necessarily verifies $\bar{J}_{\mathrm{u}, 0}<<1$ in the linear Henry biouptake regime where the coverage degree of active transporter sites by $\mathrm{M}$ is very low. The function $g$ provides a rationale for the non-linear coupling between dynamics of $\mathrm{M}$ partitioning at the interface formed between each photoactive cell sensor and the medium, and dynamics of intracellular processes leading to light emission (Figure 1C). The first term between brackets in eq 9 further highlights that the time dependence of the bioluminescence produced by the whole assembly of biosensors results from the 
non-linear interplay (convolution product) beween intertwined dynamics of the aforementioned biointerfacial and intracellular processes, and changes in concentration of photoactive biosensors over time. Literature data suggest that the quenching time $\tau_{\mathrm{q}}$ for photoactive luciferase is of the order of the minute at most, ${ }^{23}$ i.e. several orders of magnitude shorter than the typical time window over which bioluminescence is measured (several hours). ${ }^{18}$ Accordingly, for $t>>\tau_{\mathrm{q}}$ eq 9 simplifies into (SI-3)

$$
\mathcal{L} \operatorname{Lum}(\bar{t})=k_{v} V_{\mathrm{T}} \frac{k_{\mathrm{f}} \tau_{\mathrm{q}}}{\bar{K}_{\mathrm{Hi}}}\left\{f(\bar{t}) \otimes \bar{c}_{\mathrm{p}}(\bar{t})\right\}
$$

, where $f$ depends on time according to

$$
\begin{aligned}
f(\bar{t})= & \frac{\bar{J}_{\mathrm{u}, 0}}{\Lambda \bar{K}\left(1-\frac{x}{k_{\mathrm{r}} \tau_{\mathrm{a}}}+\bar{K}\left(1+x-k_{\mathrm{r}} \tau_{\mathrm{a}}\right)\right)} \times \\
& \quad\left\{\left[\frac{1}{2}\left(1-\frac{2 x}{k_{\mathrm{r}} \tau_{\mathrm{a}}}+\bar{K}(1+x)\right) \sinh (\Lambda \bar{t})-\Lambda \bar{K} \cosh (\Lambda \bar{t})\right] \mathrm{e}^{-\left(\frac{1+\bar{K}(1+x)}{2 \bar{K}}\right) \bar{t}}+\Lambda \bar{K} \mathrm{e}^{-k_{\mathrm{r}} \tau_{\mathrm{a}} \bar{t}}\right\}
\end{aligned}
$$

Equations 9-12 reveal that at metal concentrations corresponding to applicability of both the linear Henry biouptake and linear Hill regimes, $\mathcal{L} \mathrm{um}(\bar{t})$ is proportional to $\bar{J}_{\mathrm{u}, 0}$ or, equivalently, to the total metal concentration $c_{\mathrm{M}}^{*}$ regardless the magnitude of the ratio $t / \tau_{\mathrm{q}}$. Relaxing the condition $\varepsilon_{\text {ads }} \rightarrow 0$ assumed for the derivation of eqs 9-12, it can be shown that eqs 9-12 remain applicable after replacement of $\bar{c}_{\mathrm{p}}(\bar{t})$ therein by $\bar{c}_{\mathrm{p}}(\bar{t})\left(1-\varepsilon_{\mathrm{ads}} \bar{c}_{\mathrm{p}}(t)\right)$. For cases where concentration of photoactive metal-sensing cells is constant with time, $\mathcal{L} \mathrm{um}(\bar{t})$ then becomes proportional to the quadratic term $c_{\mathrm{p}}\left(1-\beta_{\mathrm{a}} S_{\mathrm{a}} \hat{K}_{\mathrm{H}} c_{\mathrm{p}}\right)$, which is in line with experimental data. ${ }^{18}$ Finally, inspection of eqs 4-6 and 8 allows to state that the conditions identified in this section that lead to a linear dependence of $\mathcal{L} u m(\bar{t})$ on $c_{\mathrm{M}}^{*}$ (at any $t$ ) are both necessary and sufficient. The non-linearity of eq 9 with respect to the time-dependent concentration of photoactive cell sensors renders impossible any tractable formulation of the ratio between $\mathcal{L}$ um $(\bar{t})$ and $c_{\mathrm{p}}(\bar{t})$. A representation of this 'bioluminescence transfer function' can be elaborated in the Laplace $s$ domain where $s$ has frequency dimension $\left(\mathrm{s}^{-1}\right)$. The reader is referred to SI-4 (Figure S-1, eqs S32,S40) for detailed discussion and physical interpretation of the biosensors response in the Laplace domain.

\section{RESULTS \& DISCUSSION}


Impacts of the dynamics of interfacial and intracellular processes operational at the single cell level on bioluminescence. We first tackle situations where passive biosorption of $\mathrm{M}$ is insignificant $\left(\varepsilon_{\mathrm{ads}} \rightarrow 0\right)$, where the concentration of photoactive biosensors is independent of time $\left(\bar{c}_{\mathrm{p}}(t)=1\right)$ and the metal concentration warrants applicability of the linear Henry biouptake regime and of the linear Hill regime (eqs 9-10).

Under such conditions, Figure 2A reports how the dimensionless bioluminescence $\mathcal{L} \operatorname{um}(\bar{t}) / \Omega$ is affected by the interplay between kinetics of $\mathrm{M}$ excretion and of $\mathrm{MP}_{\text {reg }}$ complex formation, and by the extent according to which the rate of metal biouptake is determined by $\mathrm{M}$ diffusion from the supplying medium to the biosurface. All these factors are subsumed in the dimensionless quantity $x=k_{\mathrm{e}} \tau_{\mathrm{a}} /\left(1+B n^{-1}\right)$. For $x \neq 0$, luminescence first increases with time, goes through a maximum at $\bar{t}=\bar{t}_{\max }$ and then decreases to zero with a characteristic time decay that increases upon decreasing $x$. In other terms, light production by metal-sensing cells lasts over time when metal excretion is extremely slow compared to $\mathrm{MP}_{\text {reg }}$ complex formation $\left(k_{\mathrm{e}} \tau_{\mathrm{a}}<<1\right)$ and/or when metal biouptake becomes severely limited by $\mathrm{M}$ diffusion from the bulk solution to the biosurface $\left(B n^{-1}>1\right)$, which conforms to intuitive expectation. Both situations refer to performant biosensors for which $\mathrm{M}$ efflux is not significant and/or kinetics of $\mathrm{M}$ transfer across the cell membrane is fast compared to transport of $\mathrm{M}$ from bulk solution to cell surface. In addition, the magnitude of the maximum reached by the dimensionless bioluminescence, $\mathcal{L u m}_{\max } / \Omega$, increases with decreasing $x$ (i.e. with increasing biosensors performance) and tends to the limit $\bar{J}_{\mathrm{u}, 0} \tau_{\mathrm{q}}\left[(1+\bar{K}) k_{\mathrm{r}} \tau_{\mathrm{a}}^{2}\right]^{-1}$ for $x \rightarrow 0$ (Figure 2B) (details in SI-5, eq S52 therein), where we recall that $\bar{J}_{\mathrm{u}, 0} \sim 1 /\left(1+B n^{-1}\right)$. These findings are in line with the analysis of the bioluminescence transfer function detailed in SI-4 (Figure S-1). The maximum in luminescence is shifted to larger $\bar{t}_{\max }$ with decreasing $x$ (inset Figure 2B), and it vanishes in the hypothetical limit where there is no metal leakage from intra- to extra-cellular volumes ( $x \rightarrow 0$, Figure 2A), as further discussed in SI-4. It is also found that $\bar{t}_{\max } \sim\left(k_{\mathrm{r}} \tau_{\mathrm{a}}\right)^{-1} \ln (1 / x)$ at $x<<1$ (inset Figure 2B, details in SI-5, eq S47 therein): the slower is luciferase degradation (timescale $1 / k_{\mathrm{r}}$ ) compared to $\mathrm{MP}_{\text {reg }}$ complex formation (timescale $\tau_{\mathrm{a}}$ ), the more efficient is the biosensor over time, i.e. the larger are $\bar{t}_{\max }$ and $\mathcal{L} \mathrm{Lm}_{\max } / \Omega$. These modulations of the bioluminescence signal with changing the Deborah number $1 /\left(k_{\mathrm{r}} \tau_{\mathrm{a}}\right)$ are further confirmed in Figure 2C. In the very favorable case where luciferase degradation operates at a timescale that is much larger than $\tau_{\mathrm{a}}$ 
(i.e. $\left.1 /\left(k_{\mathrm{r}} \tau_{\mathrm{a}}\right) \rightarrow \infty\right)$, it is found that $\mathcal{L u m}_{\max } / \Omega \rightarrow \bar{J}_{\mathrm{u}, 0} \tau_{\mathrm{q}} /\left(x \tau_{\mathrm{a}}\right)$ (details in SI-5, eq S53) and that bioluminescence thus becomes independent of $k_{\mathrm{r}}$. Luciferase degradation then becomes immaterial within the timescale of luminescence production, a feature that is obviously desired for achieving optimal detection of metallic ions. In this limit, Figure 2D (inset) further specifies that the rate of increase of $\bar{t}_{\max }$ with increasing $\ln \left(1 /\left(k_{\mathrm{r}} \tau_{\mathrm{a}}\right)\right)$ is solely determined by the kinetic and thermodynamic determinants of metal partitioning at the biointerface (i.e. $x$ and $\bar{K}$ ) and is given by $1 /\left(\frac{1+\bar{K}(1+x)}{2 \bar{K}}-\Lambda\right)$ (details in SI-5, eq S48 therein). Similar analysis may be performed for deciphering the increased performance of biosensors with increasing the stability constant $\bar{K}$ of metal-repressor protein $\mathrm{MP}_{\text {reg }}$ complex and increasing the quenching time $\tau_{\mathrm{q}} / \tau_{\mathrm{a}}$. The reader is referred to SI-6 (Figure S-2) for details.

The initial slope of the curves displayed in Figures 2A,C and in Figures S-2A,C (SI-6) corresponds to the maximal rate of photons production. If bioluminescence emission is not significantly limited over time by metal excretion and luciferase degradation, this slope (in counts $\mathrm{s}^{-2}$ ) reads as (details in SI-7, eq S63 therein)

$$
\mathrm{d} \mathcal{L} \operatorname{um}(t) /\left.\mathrm{d} t\right|_{t=0}=\alpha S_{\mathrm{a}} c_{\mathrm{p}}^{\max } \bar{K} J_{\mathrm{u}, 0} /(1+\bar{K})
$$

, where $\alpha=k_{v} k_{\mathrm{f}} \tau_{\mathrm{q}} V_{\mathrm{T}} / K_{\mathrm{Hi}}$ (counts $\mathrm{s}^{-1} \mathrm{~mol}^{-1} \mathrm{~m}^{3}$ ) is the luminescence yield per mole of $\mathrm{MP}_{\text {reg }}$ complex over the volume $V_{\mathrm{T}}$. Equation 13 is consistent with the expression of the maximal rate of bioluminescence emission anticipated in our earlier report. ${ }^{18}$ As an intermediate conclusion, results in Figure 2 and in Figure S-2 show that the shape of the bioluminescence peak is strongly affected by the determinants of metal partitioning at the biosensor/solution interface, by the kinetics of luciferase degradation, by the metal-repressor protein complex stability and by quenching kinetics. The reader is referred to Supporting Information (SI-8, Figure S-3) for further detailed discussion of the biosensors response in situations where non-linear metal biouptake and/or non-linear Hill regimes are operational. In particular, the analysis clarifies how the performance of the bionsensor is further connected to the magnitudes of the Hill cooperativity index $n$, of the dimensionless Hill stability constant $\bar{K}_{\mathrm{Hi}}$ and of the metal bioavailability number $\mathrm{Bn}$.

On the interplay between time-dependent concentration of photoactive metal-sensing cells and bioluminescence. We report in Figure 3 the characteristic dependences of $\operatorname{Lum}(\bar{t}) / \Omega$ on time for selected 'growth' patterns of active metal-sensing cells generated on the basis of the Gompertz model ${ }^{49}$ detailed in SI-9 (eqs S64-S66) and commonly adopted to interpret dynamics of microbial 
population. ${ }^{23,49,50}$ It is emphasized that eqs 4-6, 8 are valid for any type of time-dependent function $\bar{c}_{\mathrm{p}}(\bar{t})$ , whether it underpins decreasing or increasing photoactive cell population following cell growth/death, or changes in cell metabolism resulting from e.g. metal toxicity effects or variation of nutrients bioavailability in the medium. For the sake of demonstration, we focus here on situations where $\bar{c}_{\mathrm{p}}(\bar{t})$ increases with $\bar{t}$ according to Gompertz law for cells growth kinetics. Figure 3A shows how the bioluminescence signal is mediated by $\bar{c}_{\mathrm{p}}(\bar{t})$ in situations where (i) the initial concentration $\bar{c}_{\mathrm{p}}(0)$ of photoactive cells (which represents a fraction of the total cells number) is zero, (ii) the maximum growth rate -as reflected by the derivative $\mathrm{d} \bar{c}_{\mathrm{p}}(\bar{t}) / \mathrm{d} \bar{t}$ at the inflexion point of the growth curve- is maintained constant, and (iii) the cell population lag time is changed. The results clearly evidence that the magnitude of the bioluminescence peak corresponding to situations where $\bar{c}_{\mathrm{p}}(\bar{t}) \neq 1$ is lower than that obtained for the 'reference' case $\bar{c}_{\mathrm{p}}(t)=1$ (curve (a) in Figure 3A), its width is larger and its asymmetry is reduced. The bioluminescence peak is further detected within the time window where there is a significant increase of $\bar{c}_{\mathrm{p}}(t)$. Under the here-tested conditions, the peak is shifted to longer times with increasing lag time, while its shape remains preserved. These features are direct consequences of the convolution product involved in the definition of $\operatorname{Lum}(\bar{t})$ (eqs 9 and 11). When decreasing the maximum cell growth rate while maintaining $\bar{c}_{\mathrm{p}}(0)=0$, the magnitude of the bioluminescence peak decreases, the peak width increases noticeably and basically coincides with the time range where $\mathrm{d} \bar{c}_{\mathrm{p}}(\bar{t}) / \mathrm{d} \bar{t}$ differs from 0 (Figure 3B). These results illustrate the necessity to properly control the growth features of photoactive cells in order to achieve the desired bioluminescence signal properties (magnitude, symmetry and time of apparition).

In Figure 4, biosensors response is evaluated for situations where $\bar{c}_{\mathrm{p}}(0)$ is varied between unity (curve (a)) and 0 (curve (g), inset of Figure 4A). It is found that the bioluminescence response takes the form of two peaks for $0<\bar{c}_{\mathrm{p}}(0)<1$, a (high) short-term peak and a (ca. $10^{3}$ smaller) long-term peak (Figure 4A and Figure 4B, respectively), whereas a single peak is detected for the cases $\bar{c}_{\mathrm{p}}(0)=0$ (the only long-term peak, curve $(\mathrm{g})$ ) and $\bar{c}_{\mathrm{p}}(0)=1$ (the only short-term peak, curve (a)). Under conditions of Figure 4, kinetics of photoative cells growth is much slower than that of the processes pictured in Figure 1C operational at the single cell level. Within the timescale of these processes, $\bar{c}_{\mathrm{p}}$ may then be considered constant with time, and the short-term behavior of the bioluminescence becomes similar to that documented in Figures 2 and S-2. The shape of the short-term peak is thus basically independent of cells 
growth features and its magnitude increases with $\bar{c}_{\mathrm{p}}(0)$. Passing some delay, the growth of photoactive cells comes into play and contributes to the bioluminescence in the form of the convolution product between $\bar{c}_{\mathrm{p}}(t)$ and a function that mirrors the short-term dynamics of the interfacial and intracellular processes involved in light production (see eqs 10,12). Similarly to Figure 3B, the long-term peak magnitude, width and asymmetry are then impacted by the rate of cell growth. Obviously, the extent of separation between the peaks is tied to the magnitude of the ratio between cell population lag time and characteristic timescale of light production at the individual cell level. For cases where linear Henry metal biouptake and linear Hill regimes apply, where M passive biosorption remains negligible over time and cells growth is not affected by the presence of $\mathrm{M}$, the magnitude of each detected peak varies linearly with the metal concentration in solution. The difference between the magnitudes of the short- and longterm peaks (for cases where both peaks are observed) is connected to the difference between light quenching timescale $\tau_{\mathrm{q}}$ and time $t$ of peak apparition. Indeed, the larger is the ratio $t / \tau_{\mathrm{q}}$ compared to unity, the smaller becomes the difference between the two terms involved in eq 9 (or equivalently in eq S22, SI-3) and the more pronounced becomes the reduction in long-term bioluminescence signal (the argument holds for given cell growth kinetic features).

From the above results, it is anticipated that more than 2 bioluminescence peaks may be detected over time for a dispersion of photoactive metal biosensors whose growth properties differ in terms of lag time, maximum growth rate and/or initial cell concentration. As a first approximation, the response of such complex mixtures of metal-sensing cells may be formulated by

$$
\mathcal{L u m}(\bar{t})=\sum_{j=1}^{M} k_{v, j} V_{\mathrm{T}} \frac{k_{\mathrm{f}, j} \tau_{\mathrm{q}, j}}{\bar{K}_{\mathrm{Hi}, j}}\left\{f_{j}(\bar{t}) \otimes \bar{c}_{\mathrm{p}, j}(\bar{t})\right\}
$$

, which holds under the conditions validating the use of eqs 11-12. The index $j$ introduces a differentiation between the (bio)physical processes schemed in Figure 1C and now applying to the $j$-th

type of cell population featuring the growth properties subsumed in $\bar{c}_{\mathrm{p}, j}(\bar{t})$. Calling $M$ the number of such distinct biosensors populations, we infer from Figure 4 and eq 14 that the corresponding bioluminescence response can exhibit between $M$ and $2 \times M$ peaks depending on the initial conditions $\bar{c}_{\mathrm{p}, j}(0)$.

\section{CONFRONTATION between THEORY and EXPERIMENTS}

We report in Figure 5A the bioluminescence response measured for a dipersion $\left(V_{\mathrm{T}}=100 \mu \mathrm{l}\right)$ of cadmium-responsive Escherichia coli bioreporters constructed from the Keio knock-out mutant JW3596 (obtained from the Coli Genetic Stock Center, Yale university ${ }^{51}$ ) along the lines detailed in our previous 
report. ${ }^{18}$ Briefly, cells were engineered upon introduction of the pZNT-lux plasmid harboring a metalinducible zntA-luxCDABE gene fusion. Bioluminescence measurements were carried out over ca. $20 \mathrm{hrs}$ in 5 fold-diluted poor-metal complexing nGGM medium $(10 \mathrm{mM} \text { ionic strength, neutral } \mathrm{pH})^{18}$ at fixed optical density $\left(\mathrm{OD}_{600 \mathrm{~nm}}=0.2\right.$, verified at the start and end of the experiments) for $\mathrm{Cd}(\mathrm{II})$ concentrations in the range 0 to $22 \mathrm{nM}$ using a Lumistar Luminometer (BMG LabTechnologies, Germany). In line with the trend displayed in Figure 4 (curve (g)), the bioluminescence response under the here-examined medium conditions exhibits a single long-term peak with a maximum located at a $t_{\max }$ that slightly increases from ca. $2.4 \times 10^{4} \mathrm{~s}$ to $3.1 \times 10^{4} \mathrm{~s}$ over the range of tested bulk Cd concentrations (denoted as $c_{\mathrm{Cd}}^{*}$ ) (Figure 5A). The amplitude $\mathcal{L} u m_{\text {max }}$ of the maximum further grows linearly with $c_{\mathrm{Cd}}^{*}$ exceeding the threshold value of $\sim 5 \mathrm{nM}$, which corresponds here to the minimum metal concentration required for induction of the luminescence (Figure 5B). This linearity implies that the bioluminescence expression given by eqs 9-10, derived for the linear Henry metal biouptake regime and linear Hill regime for luciferase production, are those to be considered for testing the validity of our formalism against the experimental data. Further considering that the measurement time $t$ is well above the characteristic timescale reported for luminescence quenching $\left(\tau_{\mathrm{q}} \leq c a .30 \mathrm{~s}\right),{ }^{23,52}$ the simplified expressions 11-12 for $\mathcal{L} \operatorname{um}(t)$ are applicable for the situation of interest. It is further verified that eqs 11-12 may be recast in the simple normalized form

$$
\frac{\mathcal{L} u m(t)}{\mathcal{L u m}_{\max }}=\frac{\tilde{f}(t) \otimes \bar{c}_{\mathrm{p}}(t)}{\tilde{f}\left(t_{\max }\right) \otimes \bar{c}_{\mathrm{p}}\left(t_{\max }\right)}
$$

, where $\tilde{f}(t)$ is here defined by

$$
\begin{aligned}
\tilde{f}(t)= & \frac{\bar{K}}{\left(1-\frac{x}{k_{\mathrm{r}} \tau_{\mathrm{a}}}+\bar{K}\left(1+x-k_{\mathrm{r}} \tau_{\mathrm{a}}\right)\right)} \times \\
& \left\{\left[\frac{(\Lambda \bar{K})^{-1}}{2}\left(1-\frac{2 x}{k_{\mathrm{r}} \tau_{\mathrm{a}}}+\bar{K}(1+x)\right) \sinh \left(\Lambda t / \tau_{\mathrm{a}}\right)-\cosh \left(\Lambda t / \tau_{\mathrm{a}}\right)\right] \mathrm{e}^{-\left(\frac{1+\bar{K}(1+x)}{2 \bar{K}}\right) \frac{t}{\tau_{\mathrm{a}}}}+\mathrm{e}^{-k_{\mathrm{r}} t}\right\}
\end{aligned}
$$

In practice, (i) the half-life $1 / k_{\mathrm{r}}$ of luciferase (which is of the order of 10 minutes or longer depending on medium conditions $)^{53}$ is significantly larger than the characteristic timescale $\tau_{\mathrm{a}}(<c a .1 \mathrm{~s})^{54}$ for association of intracellular Cd with the regulatory proteins (here ZntR), (ii) the stability constant $\bar{K}$ is well above unity, which reflects the formation of strong metal-protein complexes, and (iii) the quantity 
$x=k_{\mathrm{e}} \tau_{\mathrm{a}} /\left(1+B n^{-1}\right)$ is well below unity (the typical timescale $1 / k_{\mathrm{e}}$ for excretion of Cd(II) by E. coli cells is indeed of the order of hours). ${ }^{36,37}$ In turn, eq 16 reduces to (SI-10)

$$
\tilde{f}(t)=\left(\mathrm{e}^{-k_{\mathrm{r}} t}-\frac{k_{\mathrm{eff}}}{k_{\mathrm{r}}} \mathrm{e}^{-k_{\mathrm{eff}} t}\right)\left(1-\frac{k_{\mathrm{eff}}}{k_{\mathrm{r}}}\right)^{-1}
$$

, where we introduced the overall timescale $1 / k_{\mathrm{eff}}=\bar{K} \tau_{\mathrm{a}} / x$ for the production of Cd-ZntR complex, which includes the dynamics of Cd biouptake from the solution and the excretion process kinetics (via the dimensionless term $x$ ). In cases where the half-life of luciferase is determined by the overall production kinetics of Cd-ZntR complexes (i.e. $1 / k_{\text {eff }} \sim 1 / k_{\mathrm{r}}$ ), eq 17 further simplifies into

$$
\tilde{f}(t)=\mathrm{e}^{-k_{\mathrm{r}} t}\left(1-k_{\mathrm{r}} t\right) .
$$

As detailed in SI-3 (eq S30) and SI-4, the function $\tilde{f}(t)$, proportional to $f(t)$, scales with the derivative of the function $g(t)$ that formally defines the bioluminescence peak response to a hypothetical cell concentration pulse. Therefore, $\tilde{f}(t)$ may adopt negative values (for $k_{\mathrm{r}} t>1$ ) before tending to 0 (at $k_{\mathrm{r}} t>>1$ ). Equations 15,17 (or 18 if applicable) are devoid of the unkown constants $k_{v}, k_{\mathrm{f}}$ or $\bar{K}_{\mathrm{Hi}}$ : they therefore provide a way to build a constrained analysis of the experimental data with a reduced number of adjustable parameters. In detail, eqs 15 and 17 (or 18) involve two kinetic constants, $k_{\text {eff }}$ and $k_{\mathrm{r}}$, and three additional parameters that define the Gompertz time-dependence of the concentration $\bar{c}_{\mathrm{p}}(t)$ of photoactive cells (see eq S67 in SI-9 for details). The normalized experimental data $\mathcal{L}$ um $(t) / \mathcal{L u m}_{\max }$ are given in Figure 5C for each tested metal concentration $c_{\mathrm{Cd}}^{*}$ (falling within the linear response regime of the biosensors, Figure 5B) together with the corresponding theoretical reconstructions done on the basis of eqs 15, 17 and S67 upon Levenberg-Marquardt adjustment of the 5 aforementioned parameters. It is found that the dependence of the bioluminescence on time measured over the range 6 to $22 \mathrm{nM}$ in $\mathrm{Cd}$ concentration is remarkably recovered by theory with $1 / k_{\text {eff }} \sim 1 / k_{\mathrm{r}}=30.07 \pm 3$ mins (i.e. eq 17 reduces to eq 18) and with the $\bar{c}_{\mathrm{p}}(t)$ that are reported in Figure 5D over the tested range of Cd metal concentrations. The observed shifts of the maximum in bioluminescence to larger $t_{\max }$ with increasing $c_{\mathrm{Cd}}^{*}$ are necessarily independent of the metal biouptake flux $\bar{J}_{\mathrm{u}, 0}$ (or equivalently of $c_{\mathrm{Cd}}^{*}$ ) as inferred from eqs 11-12. Accordingly, they are recovered from theory upon adjustment of the lag time involved in the Gompertz expression (see Figure 3A) that reflects here the time required for cells to adapt to increase in $c_{\mathrm{Cd}}^{*}$ (Figure 5D). Confrontation between theory and experiments reveals an intimate connection 
between kinetics of loss in luciferase activity $\left(1 / k_{\mathrm{r}}\right.$ timescale) -whose magnitude very well conforms to that found in previous studies- ${ }^{53}$ and the production kinetics $\left(1 / k_{\text {eff }}\right.$ timescale) of Cd-regulatory protein complexes that initiate the cascade of events leading to photons emission (Figure 1). Last, under the conditions examined in Figure 5A, the amount of $\mathrm{Cd}$ ions passively biosorbed is -at given $c_{\mathrm{Cd}}^{*}$ - constant over time because the total concentration of metal-sorbing cells $c_{\mathrm{p} \text {,total }}$ (including both photoactive and non-photoactive fractions) is fixed by the optical density, here constant over the whole duration of the experiments. In turn, the bioavailable metal concentration $c_{\mathrm{Cd}, \mathrm{f}}^{*}$ is directly inferred from $c_{\mathrm{Cd}}^{*}$ using eq 1 where $c_{\mathrm{p}}(t)$ should be replaced by $c_{\mathrm{p} \text {,total }}$. The applicability of the linear Henry passive biosorption regime (and thus of eq 1 ) for the system of interest was established in our previous work. ${ }^{18}$

\section{CONCLUSIONS.}

A comprehensive formalism is detailed for the evaluation of the bioluminescence produced over time by a dispersion of metal-sensing whole cell bacterial reporters. The theory deciphers quantitatively the non-linear relationships between the amplitude and shape of the bioluminescence signal emitted by the biosensors, the biophysicochemical determinants of metal partitioning dynamics between intra- and extracellular volumes, the kinetics of metal-repressor protein complex formation and ensuing activation of reporter gene, the resulting kinetics of luciferase production and rates of light emission and quenching. It is demonstrated that the expression defining the bioluminescence response involves a convolution product between the time-dependent concentration of photoactive metal-sensing cells and a temporal function representing the intricate coupling between metal biouptake dynamics and light production kinetics. Tractable analytical expressions of the bioluminescence are elaborated under practical conditions where biosensor reponse is proportional to the concentration of bioavailable metal ions in solution. The theory is further supported by experimental data collected over ca. $20 \mathrm{hrs}$ on Cd-responsive lux-based E. coli biosensors. This successful confrontation between experiments and theory demonstrates the intricate nature of the bioluminescence signal that results from the convolution product between time dependent functions reflecting the dynamics of multiscale processes from the (reporter) gene level, up to the individual cell and population scales. It further highlights the necessity to distinguish between total cell concentration and concentration of photoactive cells, the ratio of which depends on medium composition and bioavailability of nutrients (recalling that luciferase production requires energy).

It is believed that this study lays the foundations of an original conceptual framework for quantitative and physically-sound exploitation of the dependence of bioluminescence on time, and for evaluation of metal bioavailability and toxicity toward microorganisms in complex natural aqueous samples. Any 
metal-induced perturbation of biosensors physiology shall impact on their response, and this can be captured from the current formalism that explicitly integrates variations in photoactive cells concentration with time.

\section{Supporting Information.}

Glossary of (main) symbols. (SI-1) Demonstration of eq 3 (a), and defining dimensionless forms of eqs 46 and 8 (b). (SI-2) Demonstration of the expression defining the bioluminescence in the Henry metal biouptake regime (i.e. $\bar{c}_{\mathrm{M}, \mathrm{f}}^{*}<<1$ ). (SI-3) Demonstration of eqs 9-12. (SI-4) Expression for- and analysis of the transfer function between bioluminescence and concentration of photoactive biosensors, and Figure S-1. (SI-5) Evaluation of the maximum reached by the bioluminescence (Figures 2B, 2D and Figures S-2B and S-2D in SI-6). (SI-6) Analysis of the dependence of bioluminescence on the stability constant $\bar{K}$ of metal-repressor protein $\mathrm{MP}_{\text {reg }}$ complex and on the quenching time $\tau_{\mathrm{q}} / \tau_{\mathrm{a}}$, and Figure S-2.

(SI-7) Demonstration of eq 13. (SI-8) Analysis of the dependence of bioluminescence on time under conditions where non-linear metal biouptake and/or non-linear Hill regimes are operational, and Figure S-3. (SI-9) Gompertz expression for cell growth kinetics. (SI-10) Demonstration of eq 17.

\section{References.}

1. Wei, Q.; Nagi, R.; Sadeghi, K; Feng, S; Yan, E.; Ki, S. J.; Caire, R.; Tseng, D and Ozcan A. Detection and spatial mapping of mercury contamination in water samples using a smart-phone. ACS Nano 2014, 8, 1121-1129.

2. Gabarrón, M.; Zornoza, R.; Martínez-Martínez, S.; Muñoz V. A.; Faz, Á. and Acosta, J. A. Effect of land use and soil properties in the feasibility of two sequential extraction procedures for metals fractionation. Chemosphere 2018, 218, 266-272.

3. Van Leeuwen, H. P.; Town, R. M.; Buffle, J.; Cleven, R. F. M. J.; Davison, W.; Puy, J.; van Riemsdijk, W. H. and Sigg, L. Dynamic speciation analysis and bioavailability of metals in aquatic systems. Environ. Sci. Technol. 2005, 39, 8545-8556.

4. Duval, J. F. L.; Paquet, N.; Lavoie, M. and Fortin, C. Dynamics of metal partitioning at the cellsolution interface: Implications for toxicity assessment under growth inhibition conditions. Environ. Sci. Technol. 2015, 49, 6625-6636.

5. Magalhaes, D.; da Costa Marques, M. R.; Baptista, B. and Buss, D. F. Metal bioavailability and toxicity in freshwaters. Environ. Chem. Lett. 2015, 13, 69-87.

6. Angle, J. S.; Chaney, R. L. and Rhee, D. Bacterial resistance to heavy metals related to extractable and total metal concentrations in soil and media. Soil Biol. Biochem.1993, 25, 1443-1446.

7. van der Meer, J. R. and Belkin, S. Where microbiology meets microengineering: design and applications of reporter bacteria. Nat. Rev. Microbiol. 2010, 8, 511-522.

8. Saltepe, B.; Kehribar, E.S. ; Yirmibeşoğlu, S. S. S. and Şeker, U. Ö. Ş. Cellular biosensors with engineered genetic circuits. ACS Sens. 2018, 3, 13-26.

9. Grattieri, M and Minteer, S. D. Self-powered biosensors. ACS Sens. 2018, 3, 44-53.

10. Gui, Q.; Lawson, T.; Shan, S.; Yan, L.; Liu, Y. The application of whole cell-based biosensors for use in environmental analysis and in medical diagnostics. Sensors 2017, 17, 1623-1639. 
11. De Mora, K.; Joshi, N.; Balint, B. L.; Ward, F. B.; Elfick, A. and French, C. E. A pH-based biosensor for detection of arsenic in drinking water. Anal. Bioanal. Chem. 2011, 400, 1031-1039.

12. Stocker, J.; Balluch, D.; Gsell, M.; Harms, H.; Feliciano, J.; Daunert, S.; Malik, K. A. and van der Meer, J. R. Development of a set of simple bacterial biosensors for quantitative and rapid measurements of arsenite and arsenate in potable water. Environ. Sci. Technol. 2003, 37, 4743-4750

13. Cortés-Salazar, F.; Beggah, S.; van der Meer, J. R. and Girault H. H. Electrochemical As(III) wholecell based biochip sensor. Biosens. Bioelectron. 2013, 47, 237-242.

14. Tauriainen, S.; Karp, M.; Chang, W.; Virta, M. Luminescent bacterial sensor for cadmium and lead. Biosens. Bioelectron. 1998, 13, 931-938.

15. Corbisier, P.; Van der Lelie, D.; Borremans, B.; Provoost, A.; Delorenzo, V.; Brown, N. L.; Lloyd, J. R.; Hobman, J. L.; Csoregi, E.; Johansson, B. and Mattiason, B. Whole cell and protein based biosensors for the detection of biovailable heavy metal in environmental samples. Anal. Chim. Acta 1999, 387, 235244.

16. Biran, I; Babai, R.; Levcov, K.; Rishpon, J. and Ron, E. Z. Online and in situ monitoring of environmental pollutants: electrochemical biosensing of cadmium. Environ. Microbiol. 2000, 2, 285-290.

17. Jouanneau, S.; Durand, M. J.; Courcoux, P.; Blusseau, T. and Thouand, G. Improvement of the identification of four heavy metals in environmental samples by using predictive decision tree models coupled with a set of five bioluminescent bacteria. Environ. Sci. Technol. 2011, 45, 2925-2931.

18. Pagnout, C.; Present, R. M.; Billard, P.; Rotureau, E. and Duval, J. F. L. What do luminescent bacterial metal-sensors probe? Insights from confrontation between experiments and flux-based theory. Sensors and Actuators B: Chemical 2018, 270, 482-491.

19. Van Leeuwen, H. P.; Duval, J. F. L.; Pinheiro, J. P.; Blust, R. and Town, R. M. Chemodynamics and bioavailability of metal ion complexes with nanoparticles in aqueous media. Environ. Sci. Nano. 2017, 4, 2108-2133.

20. Duval, J. F. L.; Town, R. M. and van Leeuwen, H. P. Lability of nanoparticulate metal complexes at a macroscopic metal responsive (bio)interface: expression and asymptotic scaling laws. J. Phys. Chem. C 2018, 122, 6052-6065.

21. Leonard, E.M.; Marentette, J. R.; Balshine, S. and Wood, C.M. Critical body residues, MichaelisMenten analysis of bioaccumulation, lethality and behaviour as endpoints of waterborne Ni toxicity in two teleosts. Ecotoxicology 2014, 23,147-62.

22. Duval, J. F. L. and Rotureau, E. Dynamics of metal uptake by charged soft biointerphases: impacts of depletion, internalisation, adsorption and excretion. Phys. Chem. Chem. Phys. 2014, 16, 7401-7416.

23. Delle Side, D. ; Nassisi, V.; Pennetta, C. ; Alifano, P.; Di Salvo, M.; Talà, A.; Chechkin, A.; Seno, F. and Trovato, A. Bacterial bioluminescence onset and quenching: a dynamical model for a quorum sensing-mediated property. R. Soc .Open Sci. 2017, 4, 171586. DOI: 10.1098/rsos. 171586.

24. Kelly, C. J.; Hsiung, C.-J. and Lajoie, C. A. Kinetic analysis of bacterial bioluminescence. Biotechnol. Bioeng. 2003, 81, 370-378.

25. Ang, J.; Harris, E.; Hussey, B. J.; Kil, R. and McMillen, D. R. Tuning response curves for synthetic biology. ACS Synth. Biol. 2013, 2, 547-567.

26. Harms, H.; Wells, M. C. and van der Meer, J. C. Whole-cell living biosensors: are they ready for environmental application? Appl. Microbio. Biotechnol. 2006, 70, 273-280.

27. Rogers K. R. and Gerlach, C. L. Peer Reviewed: Update on Environmental Biosensors. Scientific understanding and technological development are advancing, but commercialization, with a few exceptions, has been slow. Environ. Sci. Technol. 1999, 33, 500A-506A.

28. Austin, C. M.; Stoy, W.; Su, P., Harber, M. C.; Bardill, J. P.; Hammer, B. K. and Forest, C. R. Modeling and validation of autoinducer-mediated bacterial gene expression in microfluidic environments. Biomicrofluidics. 2014, 8, 034116. DOI: 10.1063/1.4884519.

29. Leveau, J. H. and Lindow, S. E. Predictive and interpretive simulation of green fluorescent protein expression in reporter bacteria. J. Bacteriol. 2001, 183, 6752-6762. 
30. de Jong, H.; Ranquet, C.; Ropers, D.; Pinel, C. and Geiselmann, J. Experimental and computational validation of models of fluorescent and luminescent reporter genes in bacteria. BMC Systems Biology 2010, 4, 55-71.

31. Iqbal, M.; Doherty, N.; Page, A. M. L.; Qazi, S. N. A.; Ajmera, I.; Lund, P. A.; Kypraios, T.; Scott, D. J.; Hill, P. J. and Stekel, D. J. Reconstructing promoter activity from Lux bioluminescent reporters. PLoS Comput Biol. 2017, 13, e1005731. DOI: 10.1371/journal.pcbi.1005731.

32. Welham, P. A. and Stekel, D. J. Mathematical model of the Lux luminescence system in the terrestrial bacterium Photorhabdus luminescens. Mol. Biosyst. 2009, 5, 68-76.

33. Paquet, N.; Lavoie, M.; Maloney, F.; Duval, J. F. L.; Campbell, P.G.C. and Fortin, C. Cadmium accumulation and toxicity in the unicellular alga Pseudokirchneriella subcapitata: influence of metalbinding exudates and exposure time. Environ. Toxicol. Chem. 2015, 34, 1524-1532.

34. Duval, J. F. L. Dynamics of metal uptake by charged biointerphases: bioavailability and bulk depletion. Phys. Chem. Chem. Phys. 2013, 15, 7873-7888.

35. Duval, J. F. L. and Gaboriaud, F. Progress in electrohydrodynamics of soft microbial particle interphases. Curr. Opin. Colloid Interface Sci. 2010, 15, 184-195.

36. Rotureau, E.; Billard, P. and Duval, J. F. L. Evaluation of metal biouptake from the analysis of bulk metal depletion kinetics at various cell concentrations: theory and application. Environ. Sci. Technol. 2015, 49, 990-998.

37. Present, R. M.; Rotureau, E.; Billard, P.; Pagnout, C.; Sohm, B.; Flayac, J.; Gley, R.; Pinheiro, J. P. and Duval, J. F. L. Impact of intracellular metallothionein on metal biouptake and partitioning dynamics at bacterial interfaces. Phys. Chem. Chem. Phys. 2017, 19, $29114-29124$.

38. Outten, C. E.; Outten, F. W. and O'Halloran, T. V. DNA distortion mechanism for transcriptional activation by ZntR, a Zn(II)-responsive MerR homologue in Escherichia coli, J. Biol. Chem. 1999, 274, 37517-37524.

39. Frackman, S.; Anhalt, M. and Nealson, K. H. Cloning, organization, and expression of the bioluminescence genes of Xenorhabdus luminescens. J. Bacteriol. 1990, 172, 5767-5773.

40. Hajdu R.; Pinheiro, J. P.; Galceran, J. and Slaveykova, V. I. Modeling of Cd uptake and efflux kinetics in metal-resistant bacterium Cupriavidus metallidurans. Environ. Sci. Technol. 2010, 44, 45974602.

41. Duval, J. F. L. and Ohshima, H. Electrophoresis of diffuse soft particles. Langmuir 2006, 22, 35333546.

42. Michaelis, L. and Menten, M. M. The kinetics of invertin action. 1913. FEBS Lett. 2013, 587, 27122720.

43. H. P. van Leeuwen. Metal speciation dynamics and bioavailability: inert and labile complexes Environ. Sci. Technol. 1999, 33, 3743-3748.

44. Duval, J. F. L. and van Leeuwen, H. P. Rates of ionic reactions with charged nanoparticles in aqueous media. J. Phys. Chem. A 2012, 116, 6443-6451.

45. Slaveykova, V. I. and Wilkinson, K. J. Predicting the bioavailability of metals and metal complexes: critical review of the Biotic Ligand Model. Environ. Chem. 2005, 2, 9-24.

46. Jeffreys, H. and Jeffreys, B. S. The Adams-Bashforth Method. $\$ 9.11$ in Methods of Mathematical Physics, 3rd ed. Cambridge, England: Cambridge University Press, pp. 292-293, 1988.

47. Ascher, U. M. and Petzold, L. R. Computer Methods for Ordinary Differential Equations and Differential-Algebraic Equations, SIAM, Philadelphia, ISBN 0-89871-412-5, 1998.

48. Long, T.; Tu, K.; Wang, Y.; Mehta, P.; Ong, N.; Bassler, B. and Wingreen, N. Quantifying the integration of quorum-sensing signals with single-cell resolution. PLOS Biol. 2009, 7, 1-10.

49. Tjorve, K. M. C and Tjorve, E. The use of Gompertz models in growth analyses, and new Gompertzmodel approach: an addition to the unified-Richards family. PLOS One 2017, 12, e0178691. DOI: 10.1371/journal.pone.0178691.

50. Peleg, M. and Corradini, M. G. Microbial growth curves: what the models tell us and what they cannot. Crit. Rev. Food Sci. Nutr. 2011, 51, 917-945. 
51. Baba, T.; Ara, T.; Hasegawa, M.; Takai, Y.; Okumura, Y.; Baba, M.; Datsenko, K. A.; Tomita, M.; Wanner, B. L.; Mori, H. Construction of Escherichia coli K-12 in-frame, single-gene knockout mutants: the Keio collection, Mol. Syst. Biol. 2006, 2, 2006.0008.

52. Deeva, A. A.; Temlyakova, E. A.; Sorokin, A. A.; Nemtseva, E. V.; Kratasyuk, V. A. Structural distinctions of fast and slow bacterial luciferases revealed by phylogenetic analysis. Bioinformatics 2016, 32, 3053-3057.

53. Koga, K.; Harada, T.; Shimizu, H.; Tanaka, K. Bacterial luciferase activity and the intracellular redox pool in Escherichia coli. Mol. Gen. Genomics 2005, 274, 180-188.

54. Li, W.; Wang, J.; Zhang, J.; Wang, W. Molecular simulations of metal-coupled protein folding. Curr. Opin. Struct. Biol. 2015, 30, 25-31.

\section{Graphics and captions}
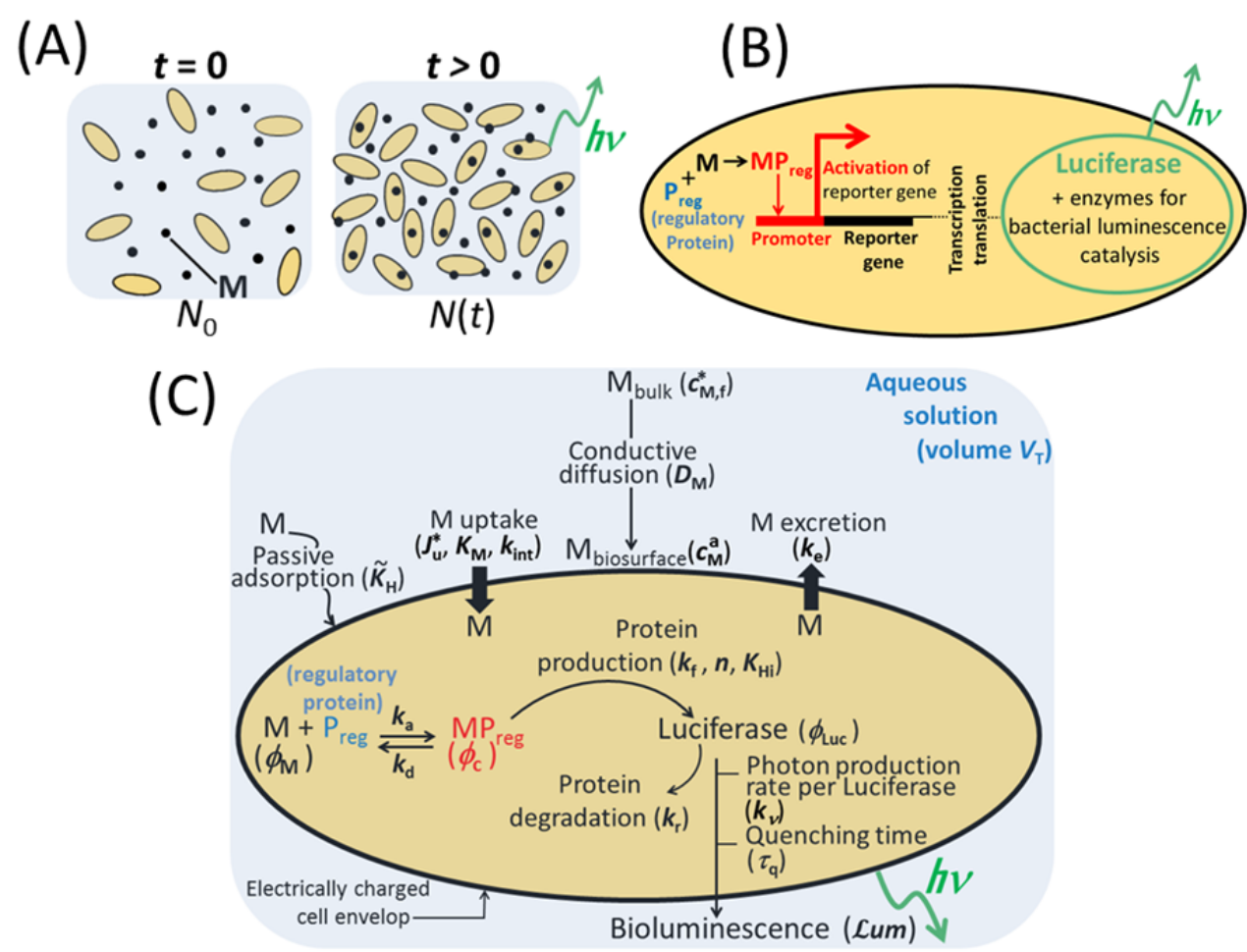

Figure 1. Extra- and intra-cellular processes involved in the chain of events leading to the production of bioluminescence at time $t$ by an assembly of whole-cell biosensors responsive to metal ions (M) and dispersed in an aqueous solution of volume $V_{\mathrm{T}}$ (A). (B) Simplified scheme of the intracellular processes controlling light emission over time. (C) Summary of the various processes and of the corresponding (main) physical parameters introduced in the theoretical formalism. 

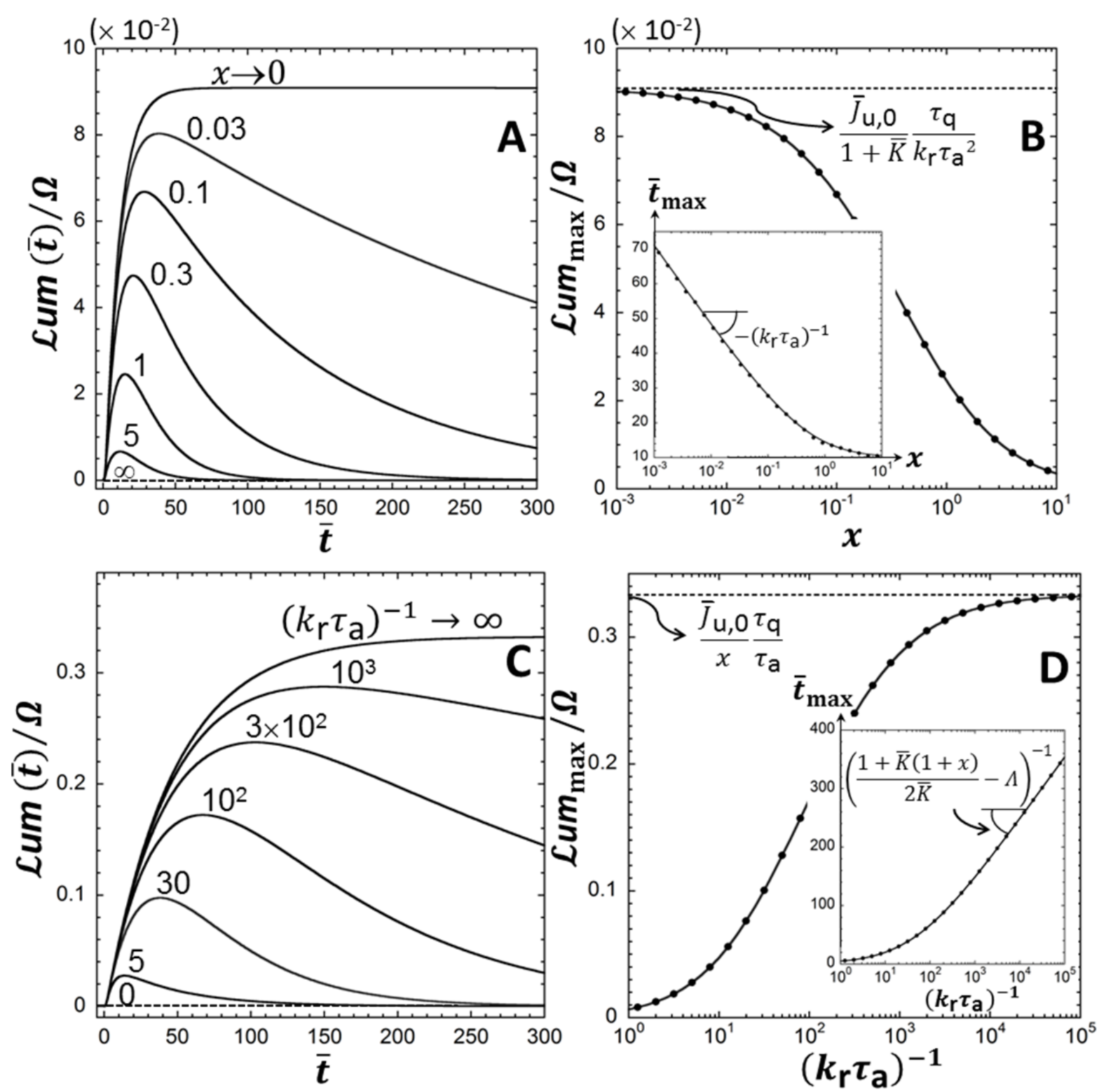

Figure 2. (A) Dependence of dimensionless bioluminescence on (dimensionless) time $\bar{t}$ for various values of $x=k_{\mathrm{e}} \tau_{\mathrm{a}} /\left(1+B n^{-1}\right)$ (indicated), and (B) corresponding features of the maximum in terms of magnitude $\left(\mathcal{L u m}_{\max } / \Omega\right)$ and position $\left(\bar{t}_{\text {max }}\right.$, in inset). (C) and (D) are the equivalents of (A) and (B) albeit for different values of $1 /\left(k_{\mathrm{r}} \tau_{\mathrm{a}}\right)$ (indicated). Computations in (A) and (C) are based on analytical eqs 9-10 or, equivalently, on numeric evaluation of eqs 2-3, 4-6, 8 taken in the linear Henry biouptake and linear Hill regimes with $\varepsilon_{\text {ads }}=0$ and $\bar{c}_{\mathrm{p}}(t)=1$. In (B) and (D) (main panel and inset): symbols derive from numerics, solid lines from the analytical expressions detailed in SI-5 (eqs S46 and S50 therein). Model parameters: $n=1, \tau_{\mathrm{q}} / \tau_{\mathrm{a}}=1, \bar{K}=10, \bar{J}_{\mathrm{u}, 0}=10^{-1}$ and $(\mathbf{A}-\mathbf{B}) 1 /\left(k_{\mathrm{r}} \tau_{\mathrm{a}}\right)=10,(\mathbf{C}-\mathbf{D}) x=0.3$. 

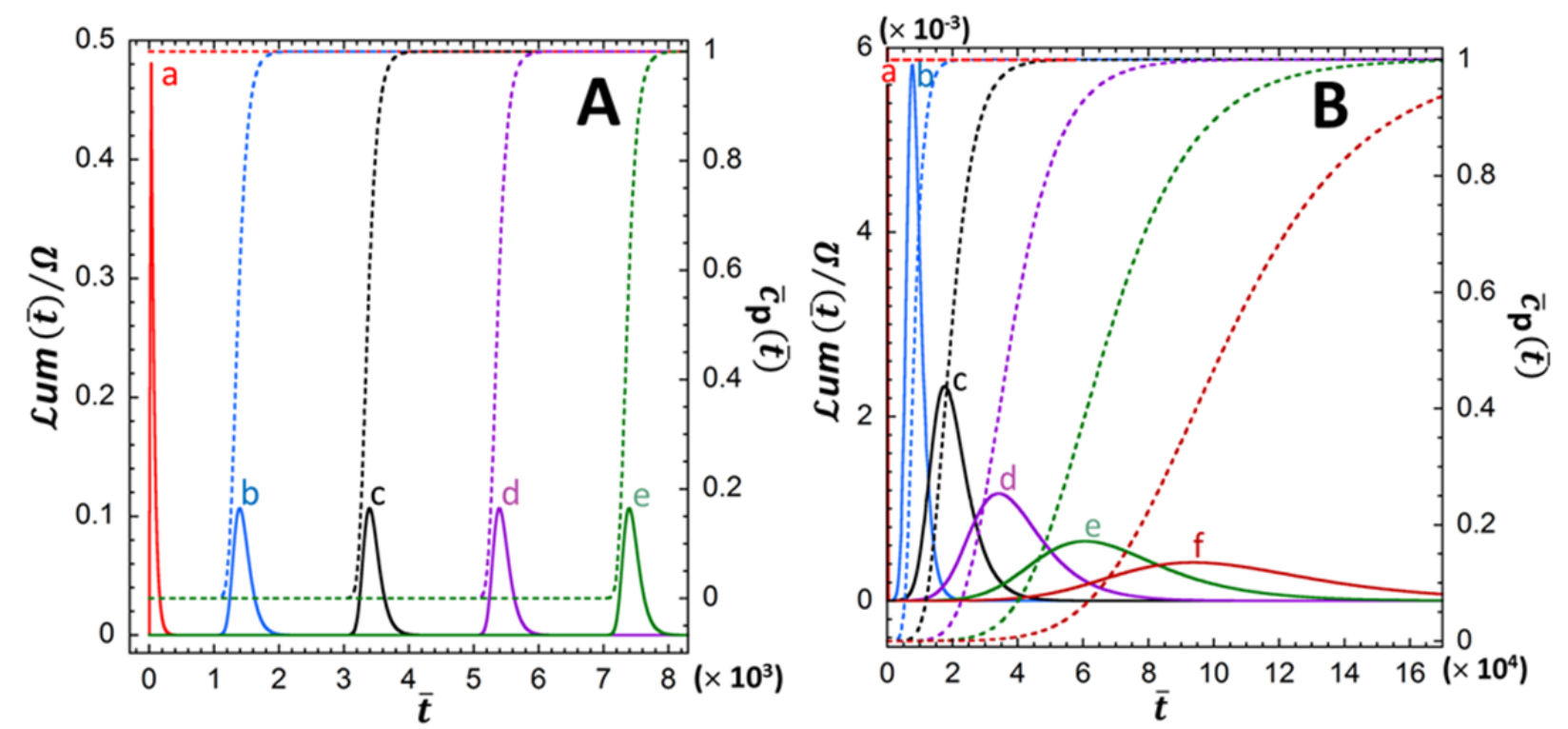

Figure 3. Dimensionless bioluminescence $\mathcal{L} \operatorname{um}(\bar{t}) / \Omega$ versus time (solid curves) for different dimensionless growth patterns $\bar{c}_{\mathrm{p}}(t)$ of active metal-sensing cells (dashed lines). (A) Impacts of the cell population lag time at constant maximum cell growth rate, (B) Impacts of maximum cell growth rate at constant lag time. Computations are based on the analytical eqs 9-10 or, equivalently, on the numeric evaluation of eqs 2-3, 4-6, 8 taken in the linear Henry biouptake and linear Hill regimes with $\varepsilon_{\text {ads }}=0$. Model parameters: $n=1, \tau_{\mathrm{q}} / \tau_{\mathrm{a}}=10, \bar{K}=10, \bar{J}_{\mathrm{u}, 0}=10^{-1}, 1 /\left(k_{\mathrm{r}} \tau_{\mathrm{a}}\right)=10, x=0.3$.

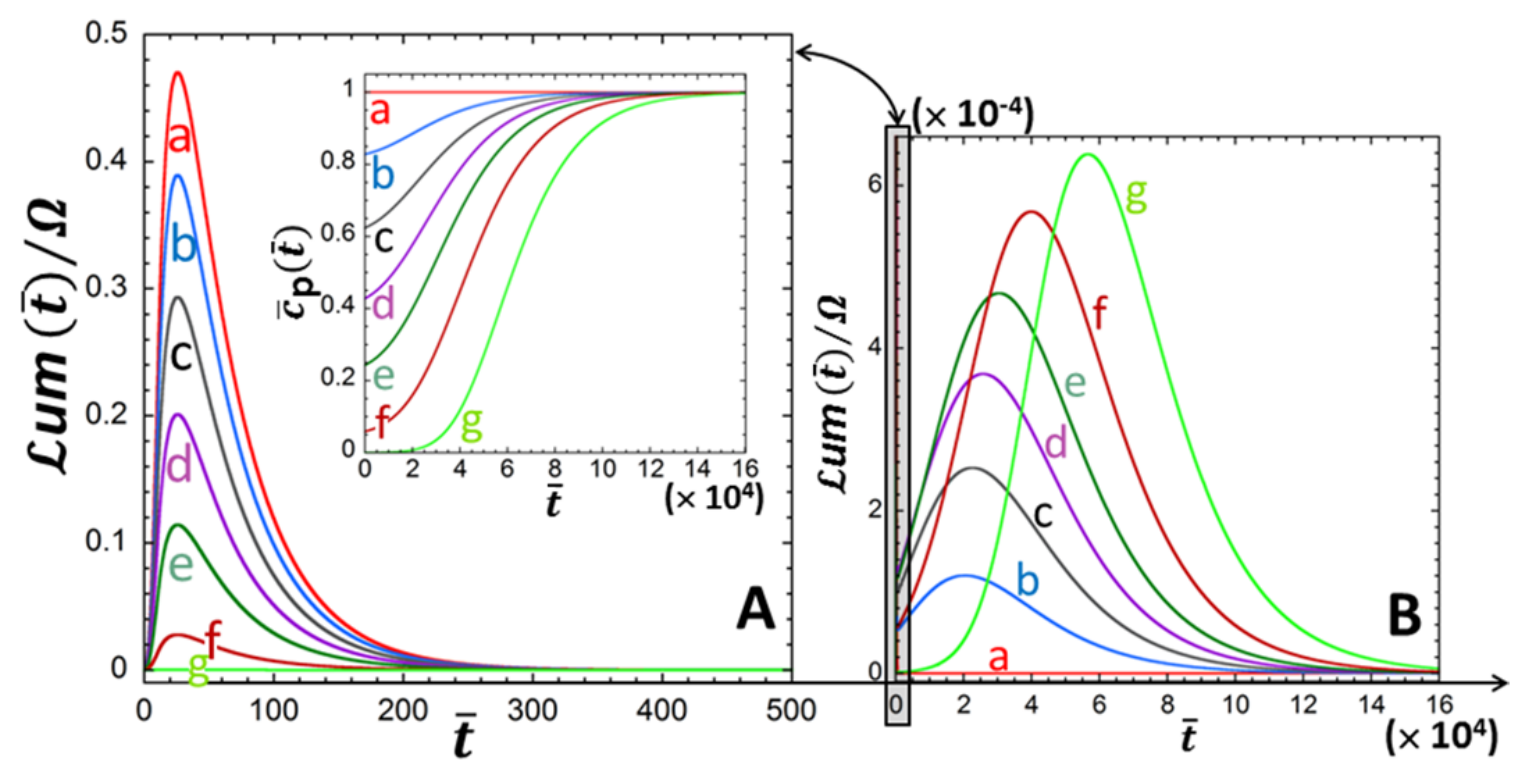

Figure 4. Dimensionless bioluminescence $\mathcal{L}$ um $(\bar{t}) / \Omega$ versus time for different dimensionless growth patterns $\bar{c}_{\mathrm{p}}(t)$ of active metal-sensing cells reported in the inset of panel A. Zoom on the short-term (A) and long-term (B) bioluminescence peaks. The magnitude of the short-term peak is $c a .10^{3}$ higher than 
that of the long-term peak. The grey-shaded time domain in panel $\mathbf{B}$ refers to the position of the short-term peak given in panel A. Computations are based on the analytical eqs 9-10 or, equivalently, on the numeric evaluation of eqs 2-3, 4-6, 8 taken in the linear Henry biouptake and linear Hill regimes with $\varepsilon_{\text {ads }}=0$. Model parameters: as in Figure 3.
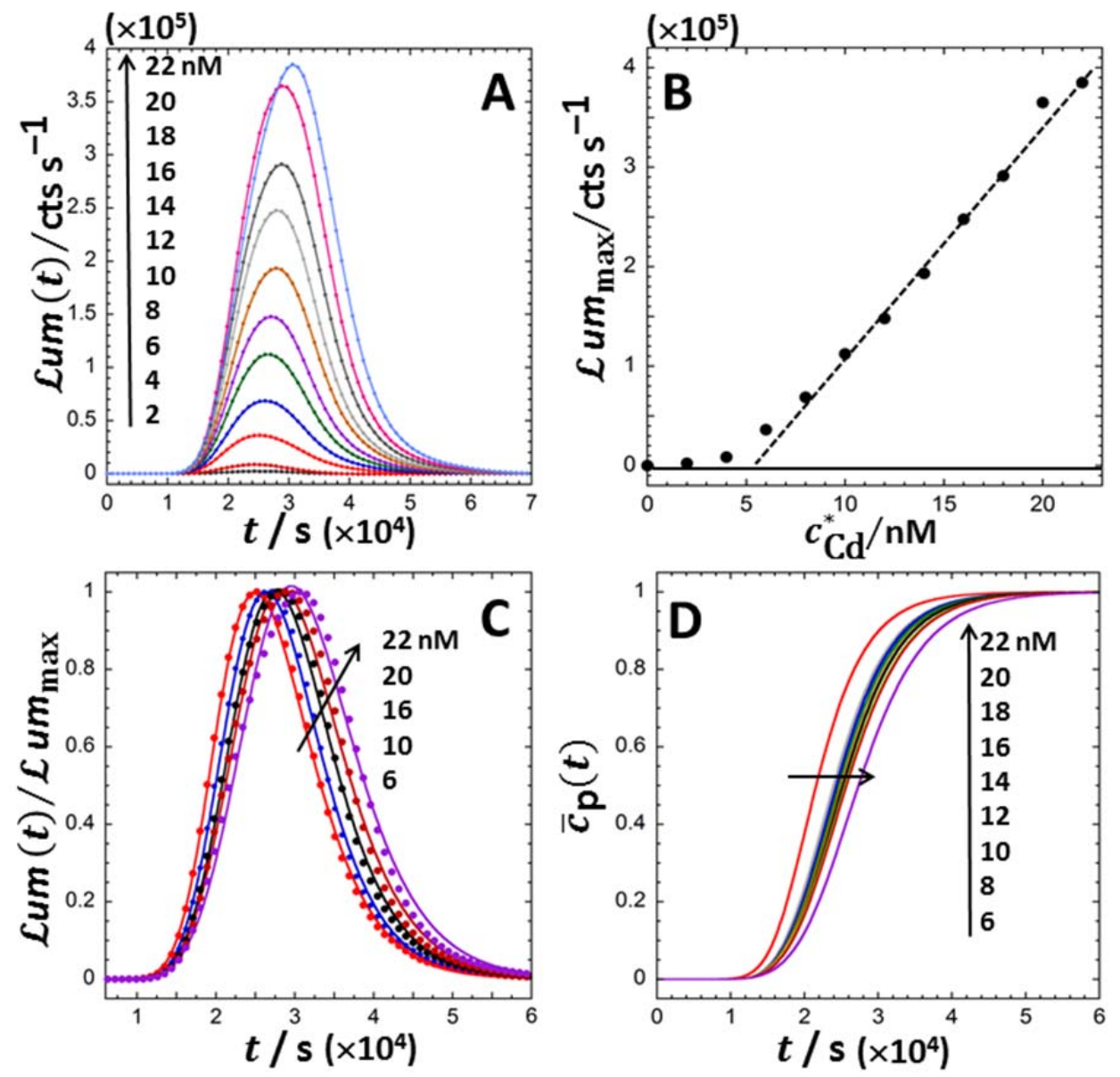

Figure 5. (A) Evolution of the bioluminescence produced by a dispersion of cadmium-responsive Escherichia coli bioreporters with time as a function of bulk concentration $\left(c_{\mathrm{Cd}}^{*}\right)$ of divalent cadmium cations (indicated). Points: experimental data. Lines: guides to the eye. Measurements were done in triplicate, experimental error $= \pm 10 \%$. (B) Dependence of the maximum $\mathcal{L}_{\max }$ in bioluminescence on $c_{\mathrm{Cd}}^{*}$. The dashed line pertains to the linear response regime. (C) Comparison between normalized bioluminescence data (points) and associated theoretical predictions (solid lines) based on eqs 15, 17 and S67 (SI-9) for various values of $c_{\mathrm{Cd}}^{*}$ (indicated). (D) Dependence of the dimensionless concentration $\bar{c}_{\mathrm{p}}(t)$ of photoactive cells on time $t$ retrieved from theory as a function of $c_{\mathrm{Cd}}^{*}$ (indicated). 


\section{TABLE OF CONTENTS graphic (TOC)}

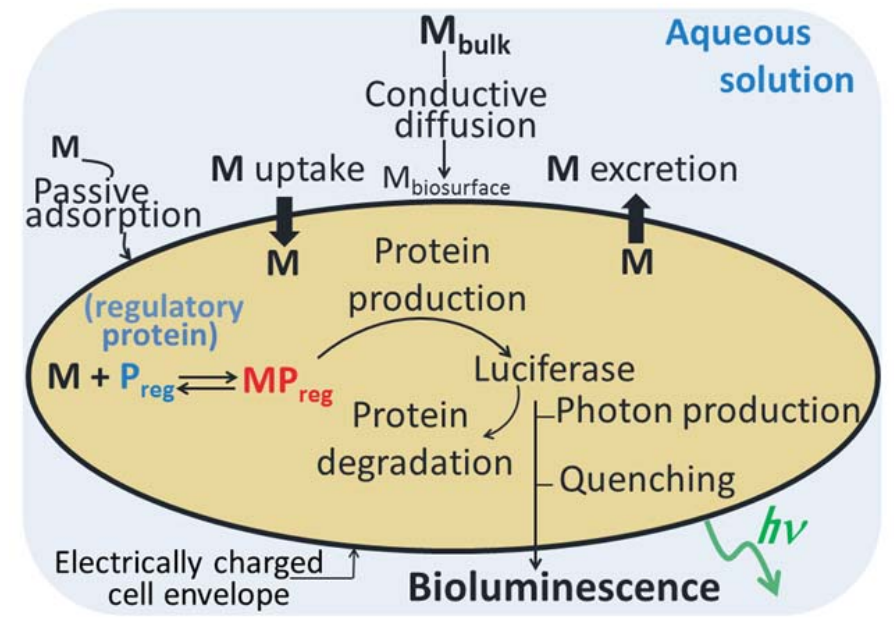




\title{
SUPPORTING INFORMATION
}

\section{Decoding the Time-Dependent Response of Bioluminescent Metal-Detecting Whole-Cell Bacterial Sensors}

\author{
Jérôme F.L. Duval, ${ }^{1, *}$ Christophe Pagnout ${ }^{2}$ \\ ${ }^{1}$ Université de Lorraine, CNRS, LIEC (Laboratoire Interdisciplinaire des Environnements Continentaux), \\ UMR7360, Vandoeuvre-lès-Nancy F-54501, France. \\ ${ }^{2}$ Université de Lorraine, CNRS, LIEC, UMR7360, Campus Bridoux, Metz F-57070, France. \\ *Corresponding author: jerome.duval@univ-lorraine.fr \\ N.B. References mentioned here correspond to those indicated in the main text. Unless otherwise \\ stated, all symbols used in this document are defined in the main text (see glossary of symbols in this \\ Supporting Information document).
}

\section{Content.}

Glossary of (main) symbols. (SI-1) Demonstration of eq 3 (a), and defining dimensionless forms of eqs 46 and 8 (b). (SI-2) Demonstration of the expression defining the bioluminescence in the Henry metal biouptake regime (i.e. $\bar{c}_{\mathrm{M}, \mathrm{f}}^{*}<<1$ ). (SI-3) Demonstration of eqs 9-12. (SI-4) Expression for- and analysis of the transfer function between bioluminescence and concentration of photoactive biosensors, and Figure S-1. (SI-5) Evaluation of the maximum reached by the bioluminescence (Figures 2B, 2D and Figures S-2B and S-2D in SI-6). (SI-6) Analysis of the dependence of bioluminescence on the stability constant $\bar{K}$ of metal-repressor protein $\mathrm{MP}_{\text {reg }}$ complex and on the quenching time $\tau_{\mathrm{q}} / \tau_{\mathrm{a}}$, and Figure S-2. (SI-7) Demonstration of eq 13. (SI-8) Analysis of the dependence of bioluminescence on time under conditions where non-linear metal biouptake and/or non-linear Hill regimes are operational, and Figure S-3. (SI-9) Gompertz expression for cell growth kinetics. (SI-10) Demonstration of eq 17. 


\section{Glossary of main symbols.}

$B n^{-1}$ Reciprocal of the bioavailability number (dimensionless).

$C_{\mathrm{p}} \quad$ Concentration of photoactive metal-sensing cells $\left(\mathrm{m}^{-3}\right)$.

$\bar{c}_{\mathrm{p}}(\bar{t})=c_{\mathrm{p}}(\bar{t}) / c_{\mathrm{p}}^{\max }$ Dimensionless concentration of photoactive metal-sensing cells.

$c_{\mathrm{M}}^{*} \quad$ Bulk metal concentration $\left(\mathrm{mol} \mathrm{m}^{-3}\right)$.

$\bar{c}_{\mathrm{M}}^{*} \quad$ Dimensionless bulk concentration of metal ions.

$c_{\mathrm{M}}^{a} \quad$ Metal concentration at the membrane surface $\left(\mathrm{mol} \mathrm{m}^{-3}\right)$.

$c_{\mathrm{M}, \mathrm{f}}^{*} \quad$ Bulk concentration of bioavailable metal ions $\left(\mathrm{mol} \mathrm{m}^{-3}\right)$.

$\bar{c}_{\mathrm{M}, \mathrm{f}}^{*} \quad$ Dimensionless bulk concentration of bioavailable metal ions.

$f \quad$ Time-dependent (dimensionless) function defined by eq 12 .

$g \quad$ Time-dependent (dimensionless) function defined by eq 10.

$J_{\text {diff }} \quad M$ diffusion flux at the membrane surface $\left(\mathrm{mol} \mathrm{m}^{-2} \mathrm{~s}^{-1}\right)$.

$J_{\mathrm{u}} \quad$ Metal biouptake flux $\left(\mathrm{mol} \mathrm{m}^{-2} \mathrm{~s}^{-1}\right)$.

$J_{\mathrm{u}}^{*} \quad$ Maximum metal biouptake flux $\left(\mathrm{mol} \mathrm{m}^{-2} \mathrm{~s}^{-1}\right)$.

$\bar{J}_{\mathrm{u}} \quad$ Dimensionless $\mathrm{M}$ biouptake flux defined by $J_{\mathrm{u}} / J_{\mathrm{u}}^{*}$.

$\bar{J}_{\mathrm{u}, 0} \quad$ Dimensionless $\mathrm{M}$ biouptake flux defined by $\bar{J}_{\mathrm{u}, 0}=\bar{c}_{\mathrm{M}}^{*} /\left(1+B n^{-1}\right)$.

$k_{\mathrm{a}} \quad$ Kinetic constant for the formation of metal-regulatory protein complexes $\left(\mathrm{mol}^{-1} \mathrm{~m}^{3} \mathrm{~s}^{-1}\right)$.

$k_{\mathrm{d}} \quad$ Kinetic constant for the dissociation of metal-regulatory protein complexes $\left(\mathrm{s}^{-1}\right)$.

$k_{\mathrm{e}} \quad$ Kinetic constant for metal excretion $\left(\mathrm{s}^{-1}\right)$.

$k_{\mathrm{f}} \quad$ Kinetic constant for luciferase production $\left(\mathrm{mol} \mathrm{m}^{-3} \mathrm{~s}^{-1}\right)$.

$k_{\text {int }} \quad$ Kinetic constant for metal internalization $\left(\mathrm{s}^{-1}\right)$.

$k_{\mathrm{r}} \quad$ Kinetic constant for luciferase degradation $\left(\mathrm{s}^{-1}\right)$.

$k_{v} \quad$ Kinetic constant for photons production per mole of luciferase molecules (counts s $\mathrm{mol}^{-1}$ ).

$K_{\mathrm{Hi}} \quad$ Hill dissociation constant between promoter and $\mathrm{MP}_{\text {reg }}$ complex $\left(\mathrm{mol} \mathrm{m}^{-3}\right)$.

$\bar{K}_{\mathrm{Hi}} \quad$ Dimensionless Hill stability constant defined by $\bar{K}_{\mathrm{Hi}}=K_{\mathrm{Hi}} /\left(J_{\mathrm{u}}^{*} \tau_{\mathrm{a}} \bar{K} S_{\mathrm{a}} c_{\mathrm{p}}^{\max }\right)$.

$K_{\mathrm{M}} \quad$ Reciprocal of the $\mathrm{M}$ affinity for the active metal transporter sites $\left(\mathrm{mol} \mathrm{m}^{-3}\right)$.

$\bar{K}=\tau_{\mathrm{d}} / \tau_{\mathrm{a}}$ Dimensionless stability constant of $\mathrm{MP}_{\text {reg }}$ complex.

$\mathcal{L} u m(t)$ Bioluminescence at time $t$ (counts $\mathrm{s}^{-1}$ ).

$\mathcal{L u m}_{\text {max }}$ Magnitude of the maximum in bioluminescence (counts $\mathrm{s}^{-1}$ ).

M Metal ion.

$\mathrm{MP}_{\text {reg }}$ Metal-regulatory protein complex.

$n \quad$ Hill cooperative binding coefficient.

$\mathrm{P}_{\text {reg }} \quad$ Regulatory (or repressor) protein.

$q \quad$ Time-dependent (dimensionless) function corresponding to light quenching (involved in eq 7).

$s \quad$ Laplace variable $\left(\mathrm{s}^{-1}\right)$.

$S_{\mathrm{a}}=4 \pi a^{2}$ Surface area of an individual cell of radius $a\left(\mathrm{~m}^{2}\right)$.

$t$ Time (s).

$\bar{t}=t / \tau_{\mathrm{a}}$ Dimensionless time. 
$\bar{t}_{\max }=t_{\max } / \tau_{\mathrm{a}}$ Dimensionless time at which bioluminescence reaches a maximum.

$V_{\mathrm{T}} \quad$ Volume of the dispersing medium $\left(\mathrm{m}^{3}\right)$.

$x \quad$ Dimensionless quantity defined by $x=k_{\mathrm{e}} \tau_{\mathrm{a}} /\left(1+B n^{-1}\right)$.

$\otimes \quad$ This symbol involved in eqs 9 and 11 stands for the convolution product operator defined by $f_{1}(\bar{t}) \otimes f_{2}(\bar{t})=\int_{0}^{\bar{t}} f_{1}(\bar{t}-\xi) f_{2}(\xi) \mathrm{d} \xi$ where $f_{1,2}(\bar{t})$ are two arbitrary time-dependent functions.

\section{Greek symbols}

$\alpha=k_{v} k_{\mathrm{f}} \tau_{\mathrm{q}} V_{\mathrm{T}} / K_{\mathrm{Hi}}$ Bioluminescence yield per mole of $\mathrm{MP}_{\text {reg }}$ over the volume $V_{\mathrm{T}}\left(\right.$ counts s $\mathrm{s}^{-1} \mathrm{~mol}^{-1} \mathrm{~m}^{3}$ ).

$\beta_{a} \quad$ Dimensionless Boltzmann accumulation factor of $\mathrm{M}$ at the charged cell envelope.

$\varepsilon_{\text {ads }} \quad$ Dimensionless parameter pertaining to passive adsorption of $\mathrm{M}$ at the biosurface (eq 1 ).

$\Lambda \quad$ Dimensionless scalar defined by eq S16 in this document.

$\tau_{\mathrm{a}}=1 /\left(k_{\mathrm{a}} \rho_{\mathrm{s}}^{V_{\mathrm{i}}}\right)$ Characteristic timescale for association of intracellular $\mathrm{M}$ with the regulatory proteins (s).

$\tau_{\mathrm{q}} \quad$ Characteristic timescale of light quenching (s).

$\tau_{\mathrm{d}}=1 / k_{\mathrm{d}}$ Timescale of dissociation of a metal-regulatory protein complex (s).

$\phi_{\mathrm{c}} \quad$ Concentration of metal-regulatory protein complexes per unit cell surface area $\left(\mathrm{mol} \mathrm{m}^{-2}\right)$.

$\phi_{\text {Luc }} \quad$ Luciferase concentration per unit cell surface area $\left(\mathrm{mol} \mathrm{m}^{-2}\right)$.

$\phi_{\mathrm{M}} \quad$ Concentration of intracellular free metal ions per unit cell surface area $\left(\mathrm{mol} \mathrm{m}^{-2}\right)$.

$\Phi_{\mathrm{M}, \mathrm{c}, \text { Luc }}$ Dimensionless concentrations of intracellular free $\mathrm{M}, \mathrm{MP}_{\text {reg }}$ complex and luciferase defined below eq 8 in the main text (subscripts $\mathrm{M}, \mathrm{c}$ and Luc, respectively).

$\Omega=k_{v} V_{\mathrm{T}} k_{\mathrm{f}} \tau_{\mathrm{a}}\left(\bar{K}_{\mathrm{Hi}}\right)^{-n}$ Prefactor in the expression of the bioluminescence (counts $\mathrm{s}^{-1}$ ), eq $\mathrm{S} 7$ in this document.

\section{SI-1.}

\section{a. Demonstration of eq 3.}

After substitution of the expressions $J_{\mathrm{diff}}(t)=\bar{f}_{\mathrm{el}} D_{\mathrm{M}}\left(c_{\mathrm{M}, \mathrm{f}}^{*}(t)-\beta_{\mathrm{a}}^{-1} c_{\mathrm{M}}^{a}(t)\right) / a \quad$ and $\bar{J}_{\mathrm{u}}(t)=J_{\mathrm{u}}(t) / J_{\mathrm{u}}^{*}=c_{\mathrm{M}}^{a}(t) /\left(K_{\mathrm{M}}+c_{\mathrm{M}}^{a}(t)\right)$ into eq 2 , it is shown that $c_{\mathrm{M}}^{a}$ is defined at any time $t$ by the quadratic equation written in the form

$$
A(1+E B)+y[1-A-B(1-E)]-y^{2}=0
$$

, with $y=c_{\mathrm{M}}^{\mathrm{a}} /\left(\beta_{a} c_{\mathrm{M}, \mathrm{f}}^{*}\right)$. The quantities $A=1 / \bar{c}_{\mathrm{M}, \mathrm{f}}^{*}$ and $B=B n^{-1} / \bar{c}_{\mathrm{M}, \mathrm{f}}^{*}$ refer to the dimensionless reciprocal of the metal affinity for the metal transporter sites and to the metal bioconversion capacity of the microorganism, respectively, ${ }^{22}$ written here in terms of $\bar{c}_{\mathrm{M}, \mathrm{f}}^{*}$ defined by $\bar{c}_{\mathrm{M}, \mathrm{f}}^{*}=\beta_{a} c_{\mathrm{M}, \mathrm{f}}^{*} / K_{\mathrm{M}}$, and of 
$B n^{-1}$. In eq S1, $E$ corresponds to the dimensionless efflux defined by $E=k_{\mathrm{e}} \phi_{\mathrm{u}} / J_{\mathrm{u}}^{*}$. The solution of eq $\mathrm{S} 1$ reads as

$$
c_{\mathrm{M}}^{\mathrm{a}} / c_{\mathrm{M}, \mathrm{f}}^{*}=\beta_{\mathrm{a}}\left\{[1-A-B(1-E)]+\left\{[A+B(1-E)-1]^{2}+4 A(1+B E)\right\}^{1 / 2}\right\} / 2 .
$$

After some rearrangements, the substitution of eq S2 into the defining expression of the metal diffusion flux $J_{\text {diff }}(t)$ leads to eq 3 in the main text. In the limit $E=0$ corresponding to the absence of metal efflux, the biouptake flux identifies with the metal diffusion flux (see eq 2). We then obtain in this limit

$$
\frac{J_{\mathrm{diff}}(t)}{J_{\mathrm{u}}^{*}}=\frac{J_{\mathrm{u}}(t)}{J_{\mathrm{u}}^{*}}=\frac{\bar{c}_{\mathrm{M}, \mathrm{f}}^{*}(t)}{2 B n^{-1}}\left(1+\frac{1+B n^{-1}}{\bar{c}_{\mathrm{M}, \mathrm{f}}^{*}(t)}\right)\left\{1-\left[1-\frac{4 B n^{-1}}{\bar{c}_{\mathrm{M}, \mathrm{f}}^{*}(t)\left(1+\frac{1+B n^{-1}}{\bar{c}_{\mathrm{M}, \mathrm{f}}^{*}(t)}\right)^{2}}\right]^{1 / 2}\right.
$$

, which correctly compares to the expression of the metal biouptake flux provided in Ref. [34] (eq 18 therein). At sufficiently low metal concentrations $\bar{c}_{\mathrm{M}, \mathrm{f}}^{*}<<1$ and under weak metal excretion conditions ( $\left.k_{\mathrm{e}} \phi_{\mathrm{M}}(t) / J_{\mathrm{u}}^{*}<<1\right), \quad$ linearization of eq $\mathrm{S} 3$ with respect to $\bar{c}_{\mathrm{M}, \mathrm{f}}^{*}$ leads to $\bar{J}_{\mathrm{u}}(\bar{t})=\frac{J_{\mathrm{u}}(t)}{J_{\mathrm{u}}^{*}}=\frac{1}{1+B n^{-1}}\left(\bar{c}_{\mathrm{M}, \mathrm{f}}^{*}(t)+k_{\mathrm{e}} \tau_{\mathrm{a}} B n^{-1} \frac{\Phi_{\mathrm{M}}(\bar{t})}{\bar{c}_{\mathrm{p}}(\bar{t})}\right)$. From that equation taken in the limit of insignificant passive biosorption of $\mathrm{M}$ (i.e. $\bar{c}_{\mathrm{M}, \mathrm{f}}^{*} \approx \bar{c}_{\mathrm{M}}^{*}$, see eq 1$)$, we infer that $\bar{J}_{\mathrm{u}}(\bar{t})$ increases linearly with increasing $\bar{c}_{\mathrm{M}}^{*}$ with a slope $1 /\left(1+B n^{-1}\right)$, in agreement with our statement below eq 3 .

\section{b. Dimensionless forms of eqs 4-6 and 8 .}

The set of coupled eqs 4-6 and 8 fully defines the time-dependent concentrations $\phi_{\mathrm{M}}(t), \phi_{\mathrm{c}}(t)$, $\phi_{\text {Luc }}(t)$ and the corresponding bioluminescence $\mathcal{L}$ um $(t)$. After some algebra, eqs 4-6 may be written in the convenient dimensionless forms

$$
\begin{gathered}
\frac{\mathrm{d} \Phi_{\mathrm{M}}(\bar{t})}{\mathrm{d} \bar{t}}=\bar{J}_{\mathrm{u}}(\bar{t}) \bar{c}_{\mathrm{p}}(\bar{t})-\left(1+k_{\mathrm{e}} \tau_{\mathrm{a}}\right) \Phi_{\mathrm{M}}(\bar{t})+\Phi_{\mathrm{c}}(\bar{t}) \\
\frac{\mathrm{d} \Phi_{\mathrm{c}}(\bar{t})}{\mathrm{d} \bar{t}}=\left[\Phi_{\mathrm{M}}(\bar{t})-\Phi_{\mathrm{c}}(\bar{t})\right] / \bar{K}
\end{gathered}
$$




$$
\frac{\mathrm{d} \Phi_{\mathrm{Luc}}(\bar{t})}{\mathrm{d} \bar{t}}=\frac{\left[\Phi_{\mathrm{c}}(\bar{t})\right]^{n}}{1+\left[\Phi_{\mathrm{c}}(\bar{t}) / \bar{K}_{\mathrm{Hi}}\right]^{n}}-k_{\mathrm{r}} \tau_{\mathrm{a}} \Phi_{\mathrm{Luc}}(\bar{t})
$$

, where we have introduced the dimensionless parameters $\bar{t}=t / \tau_{\mathrm{a}}, \Phi_{\mathrm{M}}(t)=\left(J_{\mathrm{u}}^{*} \tau_{\mathrm{a}}\right)^{-1} \phi_{\mathrm{M}}(t) \bar{c}_{\mathrm{p}}(t)$, $\Phi_{\mathrm{c}}(t)=\left(J_{\mathrm{u}}^{*} \tau_{\mathrm{d}}\right)^{-1} \phi_{\mathrm{c}}(t) \bar{c}_{\mathrm{p}}(t), \Phi_{\mathrm{Luc}}(t)=\left(\frac{S_{\mathrm{a}} c_{\mathrm{p}}^{\max } \bar{K}_{\mathrm{Hi}}{ }^{n}}{k_{\mathrm{f}} \tau_{\mathrm{a}}}\right) \phi_{\mathrm{Luc}}(t) \bar{c}_{\mathrm{p}}(t)$ and $\bar{K}_{\mathrm{Hi}}=K_{\mathrm{Hi}} /\left(J_{\mathrm{u}}^{*} \tau_{\mathrm{a}} \bar{K} S_{\mathrm{a}} c_{\mathrm{p}}^{\max }\right)$ (all parameters involved in eqs S4-S6 are defined in the main text). In eq S4, the normalized metal biouptake flux $\bar{J}_{\mathrm{u}}(\bar{t})$ corresponds to the ratio $J_{\mathrm{u}}(\bar{t}) / J_{\mathrm{u}}^{*}(\leq 1)$ with $J_{\mathrm{u}}(\bar{t})$ defined by eqs 1-3 after replacing therein $\phi_{\mathrm{M}}(t)$ and $\bar{c}_{\mathrm{M}, \mathrm{f}}^{*}$ by their expressions as a function of the dimensionless concentrations $\bar{c}_{\mathrm{p}}(\bar{t})$ and $\Phi_{\mathrm{M}}(\bar{t})$. Finally, formulating eq 8 in terms of $\Phi_{\text {Luc }}$, it comes for $\mathcal{L}$ um $(\bar{t})$

$$
\mathcal{L} \operatorname{um}(\bar{t})=\Omega\left\{\Phi_{\mathrm{Luc}}(\bar{t})-H\left(\bar{t}, \frac{\tau_{\mathrm{q}}}{\tau_{\mathrm{a}}}\right) \Phi_{\mathrm{Luc}}\left(\bar{t}-\frac{\tau_{\mathrm{q}}}{\tau_{\mathrm{a}}}\right)\right\}
$$

, with $\Omega=k_{v} V_{\mathrm{T}} k_{\mathrm{f}} \tau_{\mathrm{a}}\left(\bar{K}_{\mathrm{Hi}}\right)^{-n}$ (in counts s$\left.{ }^{-1}\right)$.

\section{SI-2. Demonstration of the expression defining the bioluminescence in the Henry metal biouptake regime (i.e.. $\bar{c}_{\mathrm{M}, \mathrm{f}}^{*}<<1$ ).}

In this regime, the metal concentration in solution is so low that the condition $\bar{c}_{\mathrm{M}, \mathrm{f}}^{*}<<1$ is satisfied. The corresponding Taylor expansion of the biouptake flux $\bar{J}_{\mathrm{u}}(\bar{t})$ (eqs 2-3) under weak excretion conditions $k_{\mathrm{e}} \phi_{\mathrm{M}}(t) / J_{\mathfrak{u}}^{*}<<1$ then leads to (see SI-1a)

$$
\bar{J}_{\mathrm{u}}(\bar{t})=\frac{1}{1+B n^{-1}}\left(\bar{c}_{\mathrm{M}, \mathrm{f}}^{*}(t)+k_{\mathrm{e}} \tau_{\mathrm{a}} B n^{-1} \frac{\Phi_{\mathrm{M}}(\bar{t})}{\bar{c}_{\mathrm{p}}(\bar{t})}\right) .
$$

Substituting eq S8 into eq S4, it comes for the net production rate of intracellular M concentration

$$
\frac{\mathrm{d} \Phi_{\mathrm{M}}(\bar{t})}{\mathrm{d} \bar{t}}=\bar{J}_{\mathrm{u}, 0}\left[1-\varepsilon_{\mathrm{ads}} \bar{c}_{\mathrm{p}}(t)\right] \bar{c}_{\mathrm{p}}(t)-(1+x) \Phi_{\mathrm{M}}(\bar{t})+\Phi_{\mathrm{c}}(\bar{t})
$$

, where we have introduced the dimensionless quantity $x=k_{\mathrm{e}} \tau_{\mathrm{a}} /\left(1+B n^{-1}\right)$ and the dimensionless metal biouptake flux $\bar{J}_{\mathrm{u}, 0}=\bar{c}_{\mathrm{M}}^{*} /\left(1+B n^{-1}\right)$. The set of eqs S5-S7 and S9 consistently defines $\mathcal{L} \mathrm{um}(\bar{t})$ in the linear Henry biouptake regime where the metal bioaccumulation flux $\bar{J}_{\mathrm{u}, 0}$ is necessarily much lower than 
unity, i.e. the coverage degree of active transporter sites by $\mathrm{M}$ is very low. The general solution of the differential eq S6 that governs the time dependence of the dimensionless luciferase concentration, $\Phi_{\text {Luc }}(\bar{t})$, is written

$$
\Phi_{\mathrm{Luc}}(\bar{t})=\mathrm{e}^{-k_{\mathrm{r}} \tau_{\mathrm{a}} \bar{t}} \int_{0}^{\bar{t}} \mathrm{e}^{k_{\mathrm{r}} \tau_{\mathrm{a}} \xi} \frac{\left[\Phi_{\mathrm{c}}(\xi)\right]^{n}}{1+\left[\Phi_{\mathrm{c}}(\xi) / \bar{K}_{\mathrm{Hi}}\right]^{n}} \mathrm{~d} \xi
$$

, where we used the boundary $\Phi_{\text {Luc }}(0)=0$. Under conditions where passive adsorption of $M$ at the biosurface is not significant ( $\varepsilon_{\mathrm{ads}} \rightarrow 0$ ) and the biouptake flux may be linearized with respect to $\bar{c}_{\mathrm{M}, \mathrm{f}}^{*}$ (eq S8), the (dimensionless) concentrations $\Phi_{\mathrm{M}}(\bar{t})$ and $\Phi_{\mathrm{c}}(\bar{t})$ are determined by the coupled differential eqs S9 and S5 with the boundaries $\Phi_{M}(0)=\Phi_{c}(0)=0$. Solving this set of differential equations in the Laplace $\bar{s}$-domain where we define the Laplace transform of any function $z$ by the integral $L_{\bar{s}}\{z(\bar{t})\}=\int_{0}^{\infty} \mathrm{e}^{-\bar{s} \times \bar{t}} z(\bar{t}) \mathrm{d} \bar{t}$ (with $\bar{s}$ a dimensionless frequency), it comes

$$
L_{\overline{\mathrm{s}}}\left\{\Phi_{\mathrm{c}}(\bar{t})\right\}=L_{\overline{\mathrm{s}}}\{h(\bar{t})\} \times L_{\overline{\mathrm{s}}}\left\{\bar{c}_{\mathrm{p}}(\bar{t})\right\}
$$

, where we have introduced the time-dependent function $h$ whose Laplace transform is given by

$$
L_{\bar{s}}\{h(\bar{t})\}=\frac{\bar{J}_{\mathrm{u}, 0}}{\bar{K}\left(\bar{s}+\bar{\omega}_{+}\right)\left(\bar{s}+\bar{\omega}_{-}\right)}
$$

, with $\bar{\omega}_{ \pm}$the characteristic (dimensionless) frequencies defined by

$$
\bar{\omega}_{ \pm}=-\frac{1+(1+x) \bar{K}}{2 \bar{K}}\left\{-1 \pm\left\{1-\frac{4 x \bar{K}}{[1+(1+x) \bar{K}]^{2}}\right\}^{1 / 2}\right\} .
$$

Taking the inverse Laplace transforms of eqs S11-S12, we obtain

$$
\Phi_{\mathrm{c}}(\bar{t})=h(\bar{t}) \otimes \bar{c}_{\mathrm{p}}(\bar{t})
$$

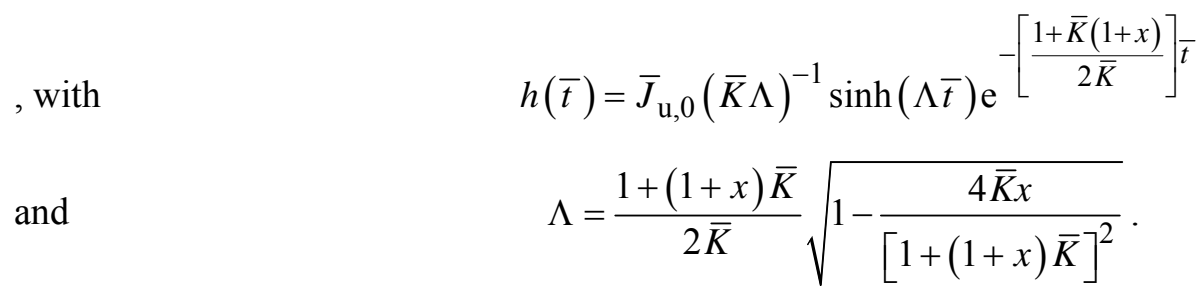

Substitution of eqs S14-S16 into eq S10 defines $\Phi_{\text {Luc }}(\bar{t})$, and further substitution of the result into eq S7 provides the expression of the bioluminescence $\mathcal{L} u m(\bar{t})$ in the Henry metal biouptake regime. 


\section{SI-3. Demonstration of eqs 9-12.}

For situations in line with applicability of the linear Hill regime for luciferase production (i.e. $n=1$ and $\left.\Phi_{\mathrm{c}}(\bar{t}) / \bar{K}_{\mathrm{Hi}}<<1\right)$, the differential eq S6 becomes

$$
\frac{\mathrm{d} \Phi_{\mathrm{Luc}}(\bar{t})}{\mathrm{d} \bar{t}}=\Phi_{\mathrm{c}}(\bar{t})-k_{\mathrm{r}} \tau_{\mathrm{a}} \Phi_{\mathrm{Luc}}(\bar{t})
$$

Further considering metal concentrations for which metal biouptake flux is defined by eq S8 (i.e. the linear Henry regime of metal biouptake is operational) and $\varepsilon_{\text {ads }} \rightarrow 0$, combination of eqs S17, S9 and S5 written in the Laplace domain $\bar{s}$ now leads to

$$
L_{\bar{s}}\left\{\Phi_{\mathrm{Luc}}(\bar{t})\right\}=L_{\overline{\mathrm{s}}}\{g(\bar{t})\} \times L_{\overline{\mathrm{s}}}\left\{\bar{c}_{\mathrm{p}}(\bar{t})\right\}
$$

, where $g$ is the function whose Laplace transform is provided by

$$
L_{\bar{s}}\{g(\bar{t})\}=\frac{\bar{J}_{\mathrm{u}, 0}}{\bar{K}\left(\bar{s}+k_{\mathrm{r}} \tau_{\mathrm{a}}\right)\left(\bar{s}+\bar{\omega}_{+}\right)\left(\bar{s}+\bar{\omega}_{-}\right)}
$$

, where $\bar{\omega}_{ \pm}$are defined by eq S13. Using eq S7, it is straightforward to show that the Laplace transform of the time-dependent bioluminescence $\operatorname{Lum}(\bar{t})$ is given by

$$
L_{\bar{s}}\{\mathcal{L u m}(\bar{t})\}=\Omega\left(1-\mathrm{e}^{-\bar{s} \tau_{\mathrm{q}} / \tau_{\mathrm{a}}}\right) L_{\overline{\mathrm{s}}}\left\{\Phi_{\mathrm{Luc}}(\bar{t})\right\}
$$

, with $\Omega=k_{v} V_{\mathrm{T}} k_{\mathrm{f}} \tau_{\mathrm{a}}\left(\bar{K}_{\mathrm{Hi}}\right)^{-1}$. Realizing from eq S18 that under the here-examined conditions $\Phi_{\mathrm{Luc}}(\bar{t})$ can be recast in the form

$$
\Phi_{\mathrm{Luc}}(\bar{t})=g(\bar{t}) \otimes \bar{c}_{\mathrm{p}}(\bar{t})
$$

, and further inverting the Laplace transform given by eq S20, it comes

$$
\mathcal{L} \operatorname{Lum}(\bar{t})=\Omega\left\{g(\bar{t}) \otimes \bar{c}_{\mathrm{p}}(\bar{t})-H\left(\bar{t}, \tau_{\mathrm{q}} / \tau_{\mathrm{a}}\right)\left[g\left(\bar{t}-\tau_{\mathrm{q}} / \tau_{\mathrm{a}}\right) \otimes \bar{c}_{\mathrm{p}}\left(\bar{t}-\tau_{\mathrm{q}} / \tau_{\mathrm{a}}\right)\right]\right\}
$$

, where the developed expression of the convolution product $g\left(\bar{t}-\tau_{\mathrm{q}} / \tau_{\mathrm{a}}\right) \otimes \bar{c}_{\mathrm{p}}\left(\bar{t}-\tau_{\mathrm{q}} / \tau_{\mathrm{a}}\right)$ is

$$
g\left(\bar{t}-\tau_{\mathrm{q}} / \tau_{\mathrm{a}}\right) \otimes \bar{c}_{\mathrm{p}}\left(\bar{t}-\tau_{\mathrm{q}} / \tau_{\mathrm{a}}\right)=\int_{0}^{\bar{t}-\tau_{\mathrm{q}} / \tau_{\mathrm{a}}} g\left(\bar{t}-\tau_{\mathrm{q}} / \tau_{\mathrm{a}}-\xi\right) \bar{c}_{\mathrm{p}}(\xi) \mathrm{d} \xi
$$

Equations S22-S23 correspond to eq 9 in the main text. The function $g$ is determined by the inverse Laplace transform of eq S19. Using eq S12, eq S19 can be written according to

$$
L_{\bar{s}}\{g(\bar{t})\}=\left(\bar{s}+k_{\mathrm{r}} \tau_{\mathrm{a}}\right)^{-1} L_{\bar{s}}\{h(\bar{t})\}
$$

, and we therefore obtain for $g$ the result 


$$
g(\bar{t})=\int_{0}^{\bar{t}} \mathrm{e}^{-k_{\mathrm{r}} \tau_{\mathrm{a}} \xi} h(\bar{t}-\xi) \mathrm{d} \xi
$$

, where $h(\bar{t})$ is defined by eq S15. Computing the integral in eq S25, after some algebraic rearrangements we derive eq 10 that explicitly defines $g(\bar{t})$ as a function of time.

In the limit $t>>\tau_{\mathrm{q}}$ (or $\bar{t} \gg>\tau_{\mathrm{q}} / \tau_{\mathrm{a}}$ ), eq S7 defining the bioluminescence can be rewritten in the form

$$
\mathcal{L u m}(\bar{t})=\Omega\left(\tau_{\mathrm{q}} / \tau_{\mathrm{a}}\right) \mathrm{d} \Phi_{\mathrm{Luc}}(\bar{t}) / \mathrm{d} \bar{t} .
$$

The Laplace transform of $\mathcal{L} u m(\bar{t})$ in this limit then becomes

$$
L_{\bar{s}}\{\mathcal{L} \operatorname{um}(\bar{t})\}=\Omega\left(\tau_{\mathrm{q}} / \tau_{\mathrm{a}}\right) s L_{\bar{s}}\left\{\Phi_{\mathrm{Luc}}(\bar{t})\right\}
$$

, where we used the boundary $\Phi_{\text {Luc }}(0)=0$. Combining eq S27 with eq S18 (which is valid in the linear Henry metal biouptake regime), it comes

$$
L_{\bar{s}}\{\mathcal{L} u m(\bar{t})\}=\Omega \times\left(\tau_{\mathrm{q}} / \tau_{\mathrm{a}}\right) \times s L_{\bar{s}}\{g(\bar{t})\} L_{\bar{s}}\left\{\bar{C}_{\mathrm{p}}(\bar{t})\right\} .
$$

In turn, the bioluminescence can be formulated according to

$$
\operatorname{Lum}(\bar{t})=\Omega \times\left(\tau_{\mathrm{q}} / \tau_{\mathrm{a}}\right) \times\left\{f(\bar{t}) \otimes \bar{c}_{\mathrm{p}}(\bar{t})\right\}
$$

, where the function $f$ verifies

$$
f(\bar{t})=\mathrm{d} g(\bar{t}) / \mathrm{d} \bar{t}
$$

, with $f(0)=g(0)=0$, which complies with the initial condition $\mathcal{L} u m(0)=0$. Equation S29 corresponds to eq 11 in the main text after realizing that $\Omega \times\left(\tau_{\mathrm{q}} / \tau_{\mathrm{a}}\right)=k_{v} V_{\mathrm{T}} k_{\mathrm{f}} \tau_{\mathrm{q}} / \bar{K}_{\mathrm{Hi}}$ for $n=1$. Using eq 10 that defines $g(\bar{t})$, eq S30 leads to eq 12 that explicitly defines the searched time-dependent function $f(\bar{t})$. The developments in SI-2 and SI-3 hold for situations where M biouptake flux varies linearily with the bioavailable metal fraction $\bar{c}_{\mathrm{M}, \mathrm{f}}^{*}$ (or $\bar{c}_{\mathrm{M}}^{*}$ for $\varepsilon_{\mathrm{ads}} \rightarrow 0$ ) and where the Hill function for luciferase production increases linearly with the concentration of intracellular metal-regulatory protein complexes (i.e. $n=1$ and $\Phi_{\mathrm{c}}(\bar{t}) / \bar{K}_{\mathrm{Hi}}<<1$ ). Applicability of both of these conditions leads to linearity between bioluminescence and $\bar{c}_{\mathrm{M}, \mathrm{f}}^{*}$, which is of paramount importance for bioanalytical detection of $\mathrm{M}$. These two conditions are both sufficient and necessary for maintaining linearity between $\mathcal{L}$ um $(\bar{t})$ and $\bar{c}_{\mathrm{M}, \mathrm{f}}^{*}$. 


\section{SI-4. Expression for- and analysis of the transfer function between bioluminescence and concentration of photoactive biosensors (Figure S-1).}

Under the conditions validating the use of eq 9, i.e. the linear Henry biouptake and Hill regimes are applicable and passive M biosorption is insignificant $\left(\varepsilon_{\text {ads }} \rightarrow 0\right.$ ), eq S20 correctly relates the Laplace transforms $L_{\bar{s}}\{\mathcal{L}$ um $(\bar{t})\}$ and $L_{\bar{s}}\left\{\Phi_{\text {Luc }}(\bar{t})\right\}$ in the dimensionless frequency domain $\bar{s}$ with $L_{\bar{S}}\left\{\Phi_{\text {Luc }}(\bar{t})\right\}$ defined by eqs S18-S19. The Laplace transform in the frequency domain $s$ simply derived from the Laplace transform in the dimensionless frequency domain $\bar{s}$ via the relationship

$$
L_{s}\{z(t)\}=\int_{0}^{\infty} \mathrm{e}^{-s \times t} z(t) \mathrm{d} t=\tau_{\mathrm{a}} L_{\bar{s}=\tau_{\mathrm{a}}}\{z(\bar{t})\}
$$

, where we used our definition of the dimensionless time $\bar{t}=t / \tau_{\mathrm{a}}$ and $z$ is a dummy time-dependent function. In turn, combining eqs S18-S20 and eq S31, we infer

$$
\bar{R}(s)=\Omega^{-1} \frac{L_{s}\{\mathcal{L} \mathrm{um}(t)\}}{L_{s}\left\{\bar{C}_{\mathrm{p}}(t)\right\}}=\bar{J}_{\mathrm{u}, 0} \frac{1-\mathrm{e}^{-\tau_{\mathrm{q}} s}}{\left(\tau_{\mathrm{a}} s+k_{\mathrm{r}} \tau_{\mathrm{a}}\right)\left(\tau_{\mathrm{a}} s+\bar{\omega}_{+}\right)\left(\tau_{\mathrm{a}} s+\bar{\omega}_{-}\right) \bar{K}}
$$

, which defines the (dimensionless) bioluminescence transfer function denoted as $\bar{R}(s)$ hereafter and where $\bar{\omega}_{ \pm}$are defined by eq S13.

If applicable, any maximum $\bar{R}\left(s_{\max }\right)$ reached by the function $\bar{R}(s)$ at the frequency $s=s_{\max }$ verifies the condition $\mathrm{d} \bar{R}(s) /\left.\mathrm{d} s\right|_{s=s_{\max }}=0$ or, equivalently, $\mathrm{d}[1 / \bar{R}(s)] /\left.\mathrm{d} s\right|_{s=s_{\max }}=0$. After some developments, we obtain

or

$$
\begin{gathered}
\tau_{\mathrm{q}} \frac{\mathrm{e}^{-\tau_{\mathrm{q}} s_{\max }}}{\mathrm{e}^{-\tau_{\mathrm{q}} s_{\max }}-1}+\frac{1}{S_{\max }+k_{\mathrm{r}}}+\sum_{i=+,-} \frac{\tau_{\mathrm{a}}}{\tau_{\mathrm{a}} s_{\max }+\bar{\omega}_{i}}=0 \\
\frac{\tau_{\mathrm{q}} \frac{\mathrm{e}^{-\tau_{\mathrm{q}} s_{\max }}}{1-\mathrm{e}^{-\tau_{\mathrm{q}} s_{\max }}}}{\frac{1}{s_{\max }+k_{\mathrm{r}}}+\sum_{i=+,-} \frac{\tau_{\mathrm{a}}}{\tau_{\mathrm{a}} s_{\max }+\bar{\omega}_{i}}}=1 .
\end{gathered}
$$

Equation S34 determines the frequency $s_{\max }$ and this expression is recalled in Figure S-1. From eq S32 taken in the limits $s \rightarrow 0$ and $s \rightarrow \infty$, we obtain the following asymptotic expressions

$$
\bar{R}(s \rightarrow 0)=\bar{J}_{\mathrm{u}, 0} \tau_{\mathrm{q}}\left(x k_{\mathrm{r}} \tau_{\mathrm{a}}\right)^{-1} s,
$$


and

$$
\bar{R}(s \rightarrow \infty)=\bar{J}_{\mathrm{u}, 0} /\left[\bar{K}\left(s \tau_{\mathrm{a}}\right)^{3}\right]
$$

Both of these limits are reported in Figure S-1. The frequency denoted as $s^{*}$ at which there is intersection between the curves associated with the low- and high-frequency behavior of $\bar{R}(s)$ (dashed lines in Figure S-1) is simply obtained by equating eqs S35 and S36. In turn, we get

$$
s^{*}=\left(\frac{x k_{\mathrm{r}}}{\bar{K} \tau_{\mathrm{q}} \tau_{\mathrm{a}}^{2}}\right)^{1 / 4} \text {. }
$$

The value taken by $\bar{R}(s \rightarrow 0)$ and $\bar{R}(s \rightarrow \infty)$ at the intersection point $s=s^{*}$ is then defined by

$$
\bar{R}^{*}=\bar{J}_{\mathrm{u}, 0} \bar{K}^{-1 / 4}\left(\frac{\tau_{\mathrm{q}}}{x k_{\mathrm{r}} \tau_{\mathrm{a}}^{2}}\right)^{3 / 4} .
$$

The point of coordinate $\left\{s^{*} ; \bar{R}^{*}\right\}$ is indicated in the log-log plot representation of $\bar{R}(s)$ given in Figure S1. In the situation $x=0$ where eq S32 is not defined, the transfer function $\bar{R}(s)$ is given by

$$
\left.\bar{R}(s)\right|_{X=0}=\frac{\bar{J}_{\mathrm{u}, 0}\left(1-\mathrm{e}^{-\tau_{\mathrm{q}} s}\right)}{\bar{K}\left(1+\tau_{\mathrm{a}} s\right)\left[\tau_{\mathrm{a}} s+\bar{K}^{-1}\left(1-\frac{1}{1+\tau_{\mathrm{a}} s}\right)\right]\left(\tau_{\mathrm{a}} s+k_{\mathrm{r}} \tau_{\mathrm{a}}\right)}
$$

, which is obtained according to the strategy adopted for deriving eq S32 albeit with substituting $x$ by 0 in eq S9. Rearrangements of eq S39 leads to

$$
\left.\bar{R}(s)\right|_{X=0}=\frac{\bar{J}_{\mathrm{u}, 0}\left(1-\mathrm{e}^{-\tau_{\mathrm{q}} s}\right)}{\tau_{\mathrm{a}} s\left(\tau_{\mathrm{a}} s+k_{\mathrm{r}} \tau_{\mathrm{a}}\right)\left[\tau_{\mathrm{a}} s+\frac{1+\bar{K}}{\bar{K}}\right] \bar{K}} .
$$

Evaluation of the low-frequency and high-frequency asymptotic limits of eq S40 then provides

and

$$
\begin{gathered}
\left.\bar{R}(s \rightarrow 0)\right|_{X=0}=\frac{\bar{J}_{\mathrm{u}, 0} \tau_{\mathrm{q}}}{(1+\bar{K}) k_{\mathrm{r}} \tau_{\mathrm{a}}^{2}}, \\
\left.\bar{R}(s \rightarrow \infty)\right|_{X=0}=\bar{J}_{\mathrm{u}, 0} /\left[\bar{K}\left(s \tau_{\mathrm{a}}\right)^{3}\right] .
\end{gathered}
$$

Equations S41-S42 correctly compare with the results indicated in Figure S-1 (blue curve therein), in particular with the absence of maximum for $\left.\bar{R}(s)\right|_{x=0}$, a feature that is further verified from analysis of the derivative of eq $\mathrm{S} 40$ with respect to $s$. 
Physically, $\bar{R}(s)$ identifies with the Laplace transform of the bioluminescence response to a hypothetical cell concentration pulse defined by $\bar{c}_{\mathrm{p}}(t) \equiv \delta(t)$ with $\delta(t)$ the Dirac function. This response in the time domain actually reads as $g(t)-H\left(t, \tau_{\mathrm{q}}\right) g\left(t-\tau_{\mathrm{q}}\right)$ where $g$ is defined by eq 10 . For cases where Henry passive M biosorption is operational (eq 1), eq S32 remains correct with $\bar{R}(s)$ then defined by $\bar{R}(s)=\Omega^{-1} L_{s}\{\mathcal{L}$ um $(t)\} / L_{s}\left\{\bar{c}_{\mathrm{p}}(\bar{t})\left(1-\varepsilon_{\text {ads }} \bar{c}_{\mathrm{p}}(t)\right)\right\}$. Figure S-1 displays in log-log plot representation the typical non-monotonous evolution of $\bar{R}$ with $s$ and evidences the presence of the maximum $\bar{R}\left(s_{\max }\right)$ reached at the frequency $s=s_{\max }$ defined by eq S34. The asymptotic ascending and descending branches of $\bar{R}$ at low and high $s$ are featured by the power laws $\sim s$ and $\sim 1 / s^{3}$, respectively, in agreement with eqs S35 and S36, respectively. The characteristic frequency $s$ * separating these asymptotic regimes is defined by eq S37 where $\bar{K}=\tau_{\mathrm{d}} / \tau_{\mathrm{a}}$ (Figure S-1). This expression of $s$ * partly reflects the complex interplay -fully captured by eq 10 in the $t$ dimension- between dynamics of interfacial and intracellular processes controlling light emission over time (see Figure 1C). In particular, we infer from that expression that performance of metal-sensing cells will last over time as quenching time increases, $\mathrm{M}$ excretion and luciferase degradation processes are sluggish or dissociation of the $\mathrm{MP}_{\text {reg }}$ complex initiating reporter gene expression is slow, which conforms to the results detailed in Figure 2 and Figure S-2. For microorganisms satisfying the condition $x=0$ (achieved e.g. for $k_{\mathrm{e}}=0$ ), the aforementioned maximum vanishes and $\bar{R}$ reduces to the frequency-independent term $\bar{J}_{\mathrm{u}, 0} \tau_{\mathrm{q}}\left[(1+\bar{K}) k_{\mathrm{r}} \tau_{\mathrm{a}}^{2}\right]^{-1}$ at $s<<s^{*}$ (eq S41), which corresponds to the long term behavior of the bioluminescence versus time (Figure 2). The situation $k_{\mathrm{e}}=0$ is ideal as it corresponds to the absence of metal leakage from intra- to extra-cellular volumes. Metal efflux constitutes however one of the homeostatic mechanisms developed by bacteria to regulate intracellular $\mathrm{M}$ concentration. Considering practical situations where $x \neq 0^{36,37}$ and assuming that the number of photoactive cells remain constant with time $\left(\bar{c}_{\mathrm{p}}(t)=1\right)$, use of eq S32 and application of the final value theorem lead to $\lim _{t \rightarrow \infty} \mathcal{L} \operatorname{um}(t)=0$. This indicates that bioluminescence systematically tends to 0 at sufficiently long time as a result of light quenching. In turn, concluding about metal toxicity from the only observation that measured bioluminescence decreases to 0 after some delay may be incorrect. Toxicity effects constitute indeed a sufficient but not necessary condition for measurement of such a bioluminescence behavior. 


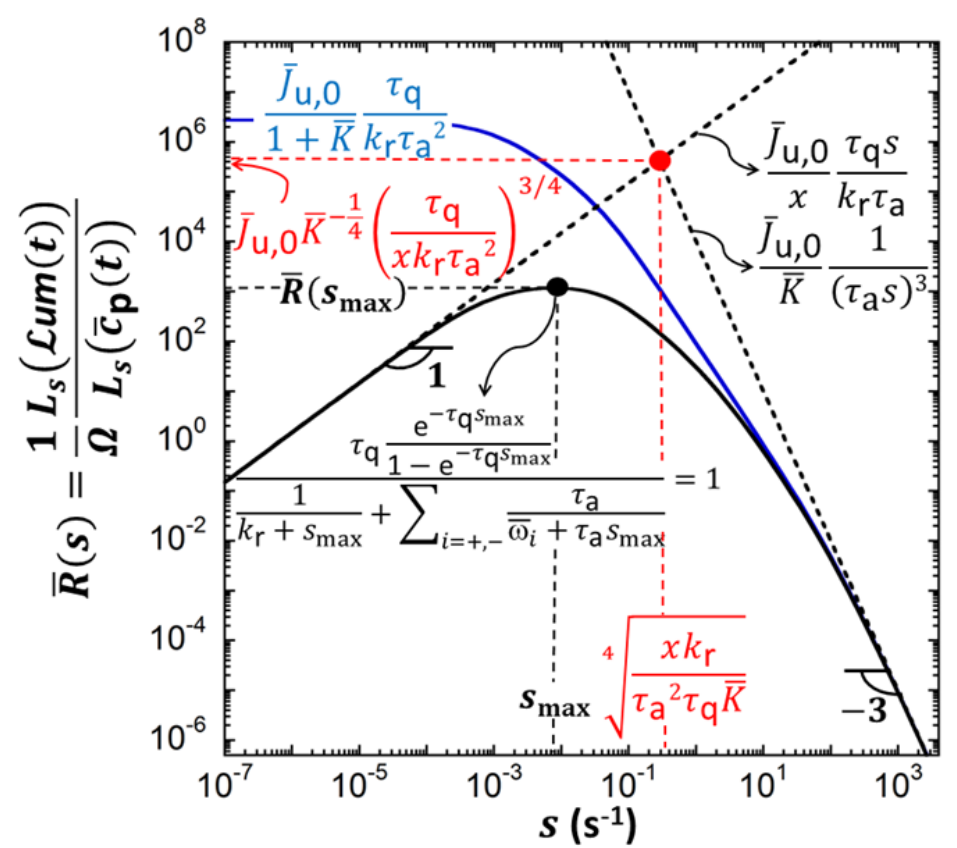

Figure S-1. Representation in the Laplace $s$-domain of the dimensionless transfer function $\bar{R}(s)$ defined by eq S32. Black solid curve: $x \neq 0$. Blue solid curve: $x=0$. Dashed lines: asymptotic behavior at $s \rightarrow 0$ and $s \rightarrow \infty$ (eqs S35-S36), the coordinates of their intersection point $\bullet$ are further given. The symbol $\bullet$ represents the maximum reached by $\bar{R}(s)$ for $x \neq 0$ with coordinates $\left\{s_{\max } ; \bar{R}\left(s_{\max }\right)\right\}$ and the implicit expression verified by $s_{\max }$ is further provided (eq S34). Model parameters: $\tau_{\mathrm{a}}=10^{-2} \mathrm{~s}, x=0.2, k_{\mathrm{r}} \tau_{\mathrm{a}}=10^{-5}, \tau_{\mathrm{q}} / \tau_{\mathrm{a}}=3 \times 10^{3}, \bar{K}=10$, $\bar{J}_{\mathrm{u}, 0}=10^{-1}$ (linear Henry biouptake regime), $n=1$ et $\bar{K}_{\mathrm{Hi}} \rightarrow \infty$ (linear Hill regime), and $\varepsilon_{\text {ads }}=0$ (insignificant M passive biosorption).

\section{SI-5. Evaluation of the maximum reached by the bioluminescence (Figures 2B, 2D and Figures S-2B and S-2D in SI-6).}

For situations where the concentration of active metal-sensing cells is independent of time $\left(\bar{c}_{\mathrm{p}}(t)=1\right)$ and the inequality $t>>\tau_{\mathrm{q}}$ is satisfied, the bioluminescence response is defined by

$$
\mathcal{L u m}(\bar{t})=k_{v} V_{\mathrm{T}} \frac{k_{\mathrm{f}} \tau_{\mathrm{q}}}{\bar{K}_{\mathrm{Hi}}} \int_{0}^{\bar{t}} f(\xi) \mathrm{d} \xi
$$

, where the function $f$ is given by eq 12. Equation S43 simply derives from eq 11 which is applicable under the conditions of Figure 2 and Figure S-2 (SI-6). Using eq S30, eq S43 simply leads to

$$
\operatorname{Lum}(\bar{t})=k_{v} V_{\mathrm{T}} \frac{k_{\mathrm{f}} \tau_{\mathrm{q}}}{\bar{K}_{\mathrm{Hi}}} g(\bar{t})
$$

, where $g$ is defined by eq 10 . The maximum in $\mathcal{L} u m(\bar{t})$, if applicable, is reached at the (dimensionless) time $\bar{t}=\bar{t}_{\text {max }}$ defined by the implicit expression $f\left(\bar{t}_{\max }\right)=0$ or equivalently 


$$
\left[\frac{1}{2}\left(1-\frac{2 x}{k_{\mathrm{r}} \tau_{\mathrm{a}}}+\bar{K}(1+x)\right) \sinh \left(\Lambda \bar{t}_{\max }\right)-\Lambda \bar{K} \cosh \left(\Lambda \bar{t}_{\max }\right)\right] \mathrm{e}^{-\left(\frac{1+\bar{K}(1+x)}{2 \bar{K}}\right) \bar{t}_{\max }}+\Lambda \bar{K} \mathrm{e}^{-k_{\mathrm{r}} \tau_{\mathrm{a}} \bar{t}_{\max }}=0
$$

, where we have used the defining expression 12 of the function $f$. Explicit analytical solutions of eq S45 may be derived in the following limits: $k_{\mathrm{r}} \tau_{\mathrm{a}} \bar{t}_{\max }>1, \Lambda \bar{t}_{\max }>1$ or $\Lambda \bar{t}_{\max }<<1$. Under the conditions tested in Figure 2 and Figure S-2, the inequality $\Lambda \bar{t}_{\max }>>1$ is verified and we then obtain for this limit

$$
\bar{t}_{\max }=\frac{1}{\frac{1+\bar{K}(1+x)}{2 \bar{K}}-k_{\mathrm{r}} \tau_{\mathrm{a}}-\Lambda} \ln \left(\frac{1}{2}-\frac{1-\frac{2 x}{k_{\mathrm{r}} \tau_{\mathrm{a}}}+\bar{K}(1+x)}{4 \Lambda \bar{K}}\right) .
$$

The solid lines in the insets of Figures 2B, 2D and S-2B correspond to predictions from eq S46.

For cases where metal efflux is not significant $(x \rightarrow 0)$, Taylor expansion of eq S46 with respect to $x$ provides

$$
\left.\bar{t}_{\max }\right|_{x \rightarrow 0}=\left(k_{\mathrm{r}} \tau_{\mathrm{a}}\right)^{-1} \ln \left\{\frac{x^{-1}(1+\bar{K})^{2}}{\left(k_{\mathrm{r}} \tau_{\mathrm{a}}\right)^{-1}+\bar{K}\left[\left(k_{\mathrm{r}} \tau_{\mathrm{a}}\right)^{-1}-1\right]}\right\} .
$$

In the limit $\left(k_{\mathrm{r}} \tau_{\mathrm{a}}\right)^{-1}>>1$, it comes from eq $\mathrm{S} 46$

$$
\left.\bar{t}_{\max }\right|_{\left(k_{\mathrm{r}} \tau_{\mathrm{a}}\right)^{-1}>>1}=\frac{1}{\frac{1+\bar{K}(1+x)}{2 \bar{K}}-\Lambda} \ln \left(\left(k_{\mathrm{r}} \tau_{\mathrm{a}}\right)^{-1} \frac{x}{2 \Lambda \bar{K}}\right) .
$$

, where it is recalled that $\Lambda$ is independent of $\left(k_{\mathrm{r}} \tau_{\mathrm{a}}\right)^{-1}$. In the extreme $\bar{K}>>1$, we get from eq S46

$$
\left.\bar{t}_{\max }\right|_{\bar{K}>>1}=\left(k_{\mathrm{r}} \tau_{\mathrm{a}}\right)^{-1} \ln \left\{\bar{K} \frac{(1+x)^{2}}{x\left[\left(k_{\mathrm{r}} \tau_{\mathrm{a}}\right)^{-1}(1+x)-1\right]}\right\} .
$$

The expressions S47, S48 and S49 conform to the slopes indicated in the insets of Figures 2B, 2D and S2B, respectively. The values taken by the dimensionless maximum in bioluminescence as displayed in Figures 2B, 2D and 3B further derive from eq S44 according to

$$
\mathcal{L u m}_{\max } / \Omega=\left(\tau_{\mathrm{q}} / \tau_{\mathrm{a}}\right) g\left(\bar{t}=\bar{t}_{\max }\right)
$$

, with $\bar{t}_{\max }$ defined by eq S46.The solid curves displayed in Figures 2B, 2D and S-2B refer to predictions obtained from eq S50.

In the limit $x \rightarrow 0$, after some rearrangements (see details in SI-7, eq S59) eqs S43-S44 reduce to 


$$
\mathcal{L} \operatorname{um}(t)=\Omega \bar{J}_{\mathrm{u}, 0} \frac{\tau_{\mathrm{q}}}{\tau_{\mathrm{a}}{ }^{2}}\left[\frac{1}{1+\bar{K}\left(1-k_{\mathrm{r}} \tau_{\mathrm{a}}\right)}\right]\left\{\frac{1-\mathrm{e}^{-k_{\mathrm{r}} t}}{k_{\mathrm{r}}}+\gamma\left(\mathrm{e}^{-t / \gamma}-1\right)\right\}
$$

, where we introduced $\gamma=\tau_{\mathrm{a}} \frac{\bar{K}}{1+\bar{K}}$. In this limit, the maximum in bioluminescence is reached for $t \rightarrow \infty$ so that

$$
\mathcal{L u m}_{\max } /\left.\Omega\right|_{X \rightarrow 0}=\frac{\bar{J}_{\mathrm{u}, 0}}{1+\bar{K}} \frac{\tau_{\mathrm{q}}}{k_{\mathrm{r}} \tau_{\mathrm{a}}^{2}} .
$$

, which corresponds to the limit provided in Figure 2B and Figure S-1 (blue curve therein). Following a similarly strategy, i.e. after simplification of eqs S43-S44 for $1 /\left(k_{\mathrm{r}} \tau_{\mathrm{a}}\right)>>1$ or $\bar{K}>>1$ and computation of the ensuing expressions at $\bar{t} \rightarrow \infty$, we derive

$$
\begin{gathered}
\mathcal{L u m}_{\max } /\left.\Omega\right|_{\left(k_{\mathrm{r}} \tau_{\mathrm{a}}\right)^{-1}>>1}=\frac{\bar{J}_{\mathrm{u}, 0}}{x} \frac{\tau_{\mathrm{q}}}{\tau_{\mathrm{a}}}, \\
\mathcal{L u m}_{\max } /\left.\Omega\right|_{\bar{K}>1}=\frac{\bar{J}_{\mathrm{u}, 0}}{1+x} \frac{\tau_{\mathrm{q}}}{k_{\mathrm{r}} \tau_{\mathrm{a}}{ }^{2}} .
\end{gathered}
$$

, which are the limits provided in Figures 2D and S-2B, respectively.

Let us now specifically analyze the situation investigated in Figure S-2D. In the limit $t>>\tau_{\mathrm{q}}$ (or $\bar{t} \gg>\tau_{\mathrm{q}} / \tau_{\mathrm{a}}$ ), the bioluminescence defined by eq S44 is proportional to $\tau_{\mathrm{q}}$, having in mind that the function $g$ is independent of the quenching time (eq 10). In turn, the time $\bar{t}=\bar{t}_{\max }$ at which the maximum in $\mathcal{L} \operatorname{um}(\bar{t})$ is reached, is independent of $\tau_{\mathrm{q}} / \tau_{\mathrm{a}}$ providing that the condition $\bar{t}_{\max } \gg \tau_{\mathrm{q}} / \tau_{\mathrm{a}}$ is respected. This feature corresponds to curve (c) in the inset of Figure S-2D with $\bar{t}_{\max }$ being defined by eq S46. The corresponding dimensionless maximum in bioluminescence is simply obtained from eq S50 (curve (a) in Figure S-2D). In the other extreme $t<<\tau_{\mathrm{q}}$ (or $\bar{t}<<\tau_{\mathrm{q}} / \tau_{\mathrm{a}}$ ), the maximum is located at $\bar{t}_{\max } \sim \tau_{\mathrm{q}} / \tau_{\mathrm{a}}$ (curve (d) in the inset of Figure S-2D) and its value is now determined by

$$
\mathcal{L u m}_{\max } / \Omega=\int_{0}^{\tau_{\mathrm{q}} / \tau_{\mathrm{a}}} g(\xi) \mathrm{d} \xi
$$

, where $g$ is defined by eq 10. Equation S55 simply derives from eq 9 taken at $\bar{t}=\bar{t}_{\max } \sim \tau_{\mathrm{q}} / \tau_{\mathrm{a}}$ in the limit $t<<\tau_{\mathrm{q}}$ (under the here examined condition $\bar{c}_{\mathrm{p}}(t)=1$ ). Curve (b) in Figure S-2D is obtained from eq S55. From the latter equation, it is further verified that 


$$
\mathcal{L} \operatorname{um}_{\max } /\left.\Omega\right|_{\tau_{\mathrm{q}} / \tau_{\mathrm{a}} \rightarrow \infty}=\int_{0}^{\infty} g(\xi) \mathrm{d} \xi
$$

, which is the result indicated in the main text.

\section{SI-6. Analysis of the dependence of bioluminescence on the stability constant $\bar{K}$ of metal- repressor protein $\mathrm{MP}_{\text {reg }}$ complex and on the quenching time $\tau_{\mathrm{q}} / \tau_{\mathrm{a}}$ (Figure S-2).}

Figure S-2A displays the dependence of bioluminescence on the stability constant $\bar{K}$ of metalrepressor protein $\mathrm{MP}_{\text {reg }}$ complex. Since the developed expression of the constant $\Omega$ involved in eq 9 is $\Omega=\left(k_{v} V_{\mathrm{T}} k_{\mathrm{f}} \tau_{\mathrm{a}}^{2} K_{\mathrm{Hi}}^{-1} J_{\mathrm{u}}^{*} S_{\mathrm{a}} c_{\mathrm{p}}^{\max }\right) \bar{K}$, this dependence is fully captured by analysis of $\bar{K} \mathcal{L} \mathrm{um}(\bar{t}) / \Omega$. Qualitatively, the results are analogous to those obtained in Figure 2C when examining the effects of $1 /\left(k_{\mathrm{r}} \tau_{\mathrm{a}}\right)$. Namely, increasing $\bar{K}$ ensures that $\mathrm{MP}_{\text {reg }}$ dissociation gradually becomes insignificant so that reporter gene activation can be efficiently initiated when a given metal ion enters the intracellular volume and associates with $\mathrm{P}_{\text {reg }}$ to form a stable $\mathrm{MP}_{\text {reg }}$ complex. In turn, $\bar{K} \mathcal{L} \operatorname{um}(\bar{t}) / \Omega$ increases with $\bar{K}$ and, doing so, the maximum $\bar{K} \mathcal{L u m}_{\text {max }} / \Omega$ is shifted to larger $\bar{t}_{\max }$ (inset Figure S-2B), as it did with increasing biosensor preformance via reducing $x$ or increasing $1 /\left(k_{\mathrm{r}} \tau_{\mathrm{a}}\right)$ (Figure 2). The magnitude of the $\mathrm{MP}_{\text {reg }}$ stability constant thus also inherently defines the efficiency with which biosensors detect metal ions. For very strong $\mathrm{MP}_{\text {reg }}$ complexes $(\bar{K} \rightarrow \infty)$, which corresponds to ideal $\mathrm{M}$ biosensing situations, $\bar{K} \mathcal{L u m}_{\max } / \Omega$ becomes independent of $\bar{K}$ (Figure S-2B) and tends to $\bar{J}_{\mathrm{u}, 0} \tau_{\mathrm{q}}\left[(1+x) k_{\mathrm{r}} \tau_{\mathrm{a}}^{2}\right]^{-1}$, while $\bar{t}_{\max }$ increases with $\bar{K}$ according to $\bar{t}_{\max } \sim\left(k_{\mathrm{r}} \tau_{\mathrm{a}}\right)^{-1} \ln \bar{K}$ (details in SI-5, eqs S49 and S54). Finally, Figure S-2C reports the effects of the (dimensionless) quenching time $\tau_{\mathrm{q}} / \tau_{\mathrm{a}}$ on bioluminescence. In the extreme where light quenching is insignificant $\left(\tau_{\mathrm{q}} / \tau_{\mathrm{a}} \rightarrow \infty\right), \mathcal{L} \mathrm{um}(\bar{t}) / \Omega$ continuously increases with $\bar{t}$ and reaches at sufficiently large $\bar{t}$ a plateau whose magnitude is $\int_{0}^{\infty} g(\xi) \mathrm{d} \xi$ (details in SI-5, eq S56 therein). This ideal metal-biosensing situation corresponds to that where any produced luciferase remains photoactive over the whole duration of luminescence acquisition, which explains the absence of maximum for the bioluminescence signal over time. For finite (and thus practical) values of $\tau_{\mathrm{q}} / \tau_{\mathrm{a}}$ and at sufficiently short time, $\mathcal{L} \operatorname{um}(\bar{t}) / \Omega$ identifies with the response evaluated at $\tau_{\mathrm{q}} / \tau_{\mathrm{a}} \rightarrow \infty$. It then reaches a maximum at $\bar{t}_{\max }$ before leveling off to zero for $\bar{t} \rightarrow \infty$. The magnitude of the maximum describes a 
sigmoidal dependence on $\tau_{\mathrm{q}} / \tau_{\mathrm{a}}$ (Figure S-2D), expressions of which can be formulated in the limits $t_{\max } / \tau_{\mathrm{q}}>>1$ and $t_{\max } / \tau_{\mathrm{q}}<<1$ (curves (a) and (b) in Figure S-2D, respectively) as extensively detailed in SI-5 (eqs S50, S55 therein).
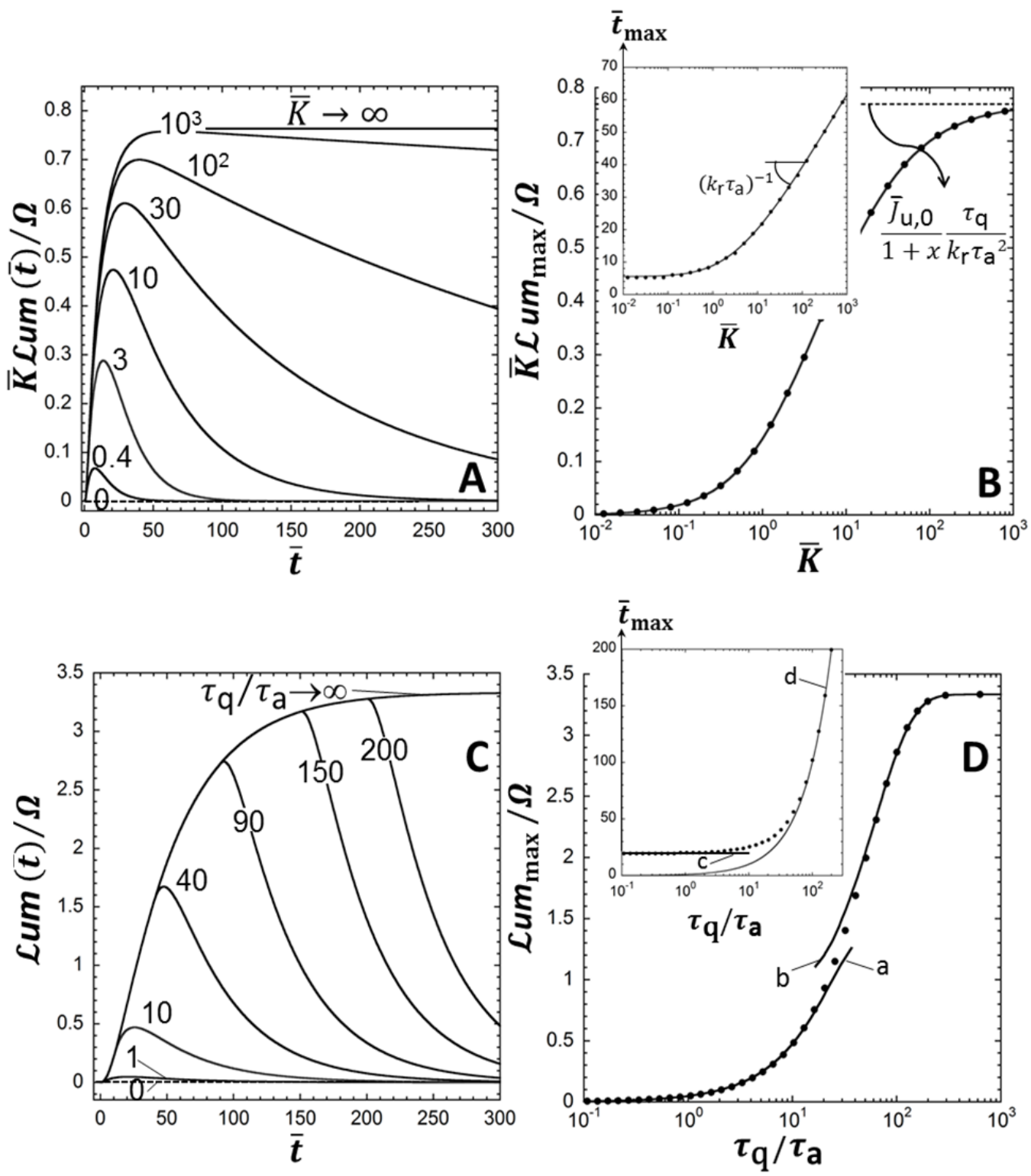

Figure S-2. Dependence of dimensionless bioluminescence on $\bar{t}$ for various values of (A) $\bar{K}$, and (C) $\tau_{\mathrm{q}} / \tau_{\mathrm{a}}$. The corresponding features of the maximum in terms of magnitude ( $\mathcal{L u m}_{\max } / \Omega$ ) and position ( $\bar{t}_{\text {max }}$, in inset) are given in (B) and (D), respectively. Computations in (A) and (C) are based on analytical eqs 9-10 or, equivalently, on numeric evaluation of eqs 2-3, 4-6, 8 taken in the linear Henry biouptake and linear Hill regimes with $\varepsilon_{\text {ads }}=0$ and $\bar{c}_{\mathrm{p}}(t)=1$. In (B) and (D) (main panel and inset): symbols derive from numerics. Solid lines in panel (B) (and inset therein) from eqs S46, S50. Solid lines in panel (D) from eq S50 (curve (a)), eq S55 (curve (b)), eq S46 (curve (c)), and $t_{\max }=\tau_{\mathrm{q}}$ (curve (d)). Other model parameters: as in Figure 2 in the main text. 


\section{SI-7. Demonstration of eq 13.}

Under the condition $t>>\tau_{\mathrm{q}}$ and within the linear Henry bioupake and Hill regimes of metal concentrations, the bioluminescence is expressed by eqs 11-12 where the function $f(\bar{t})$ becomes in the limit $x<<1$

$$
f(\bar{t})=\frac{\bar{J}_{\mathrm{u}, 0}}{1+\bar{K}\left(1-k_{\mathrm{r}} \tau_{\mathrm{a}}\right)}\left\{[\sinh (\Lambda \bar{t})-\cosh (\Lambda \bar{t})] \mathrm{e}^{-\Lambda \bar{t}}+\mathrm{e}^{-k_{\mathrm{r}} \tau_{\mathrm{a}} \bar{t}}\right\}
$$

, where $\Lambda$ is defined in the main text below eq 10 (or by eq S16) and it reduces to $\Lambda=\frac{1+\bar{K}}{2 \bar{K}}$ for $x<<1$. Simplifications of eq S57 leads to

$$
f(\bar{t})=\frac{\bar{J}_{\mathrm{u}, 0}}{1+\bar{K}\left(1-k_{\mathrm{r}} \tau_{\mathrm{a}}\right)}\left(\mathrm{e}^{-k_{\mathrm{r}} \tau_{\mathrm{a}} \bar{t}}-\mathrm{e}^{-\frac{1+\bar{K}}{\bar{K}} \bar{t}}\right) .
$$

After some algebra, the substitution of eq S58 into eq 11 yields the following expression of the bioluminescence for $\bar{c}_{\mathrm{p}}(\bar{t})=1$

$$
\operatorname{Lum}(\bar{t})=k_{v} V_{\mathrm{T}} \frac{k_{\mathrm{f}} \tau_{\mathrm{q}}}{\bar{K}_{\mathrm{Hi}}}\left[\frac{\bar{J}_{\mathrm{u}, 0}}{1+\bar{K}\left(1-k_{\mathrm{r}} \tau_{\mathrm{a}}\right)}\right]\left\{\frac{1-\mathrm{e}^{-k_{\mathrm{r}} \tau_{\mathrm{a}} \bar{t}}}{k_{\mathrm{r}} \tau_{\mathrm{a}}}+\frac{\gamma}{\tau_{\mathrm{a}}}\left(\mathrm{e}^{-\frac{1+\bar{K}_{\bar{K}}}{\bar{t}}}-1\right)\right\}
$$

, which is identical to eq S51 after realizing that $k_{v} V_{\mathrm{T}} \frac{k_{\mathrm{f}} \tau_{\mathrm{q}}}{\bar{K}_{\mathrm{Hi}}}=\Omega \frac{\tau_{\mathrm{q}}}{\tau_{\mathrm{a}}}$ and $\bar{t}=t / \tau_{\mathrm{a}}$. Taylor expansion of eq $\mathrm{S} 59$ at $\bar{t} \rightarrow 0$ results in

$$
\left.\operatorname{Lum}(\bar{t})\right|_{\bar{t} \rightarrow 0}=k_{v} V_{\mathrm{T}} \frac{k_{\mathrm{f}} \tau_{\mathrm{q}}}{\bar{K}_{\mathrm{Hi}}}\left[\frac{\bar{J}_{\mathrm{u}, 0}}{1+\bar{K}\left(1-k_{\mathrm{r}} \tau_{\mathrm{a}}\right)}\right]\left(\bar{t}-\frac{\gamma}{\tau_{\mathrm{a}}}\right) .
$$

In turn, considering the further limit $1 /\left(k_{\mathrm{r}} \tau_{\mathrm{a}}\right)>>1$, eq S60 simplifies into

$$
\operatorname{Lum}(\bar{t})=k_{\nu} V_{\mathrm{T}} \frac{k_{\mathrm{f}} \tau_{\mathrm{q}}}{\bar{K}_{\mathrm{Hi}}} \frac{\bar{J}_{\mathrm{u}, 0}}{1+\bar{K}}\left(\bar{t}-\frac{\gamma}{\tau_{\mathrm{a}}}\right)
$$

Substituting the expression $\bar{K}_{\mathrm{Hi}}=K_{\mathrm{Hi}} /\left(J_{\mathrm{u}}^{*} \tau_{\mathrm{a}} \bar{K} S_{\mathrm{a}} c_{\mathrm{p}}^{\max }\right)$ into eq S61, it comes

$$
\mathcal{L} \operatorname{Lum}(t)=k_{v} V_{\mathrm{T}} \frac{k_{\mathrm{f}} \tau_{\mathrm{q}}}{K_{\mathrm{Hi}}} S_{\mathrm{a}} c_{\mathrm{p}}^{\max } \frac{\bar{K} J_{\mathrm{u}, 0}}{1+\bar{K}}(t-\gamma)
$$

, with $J_{\mathrm{u}, 0}=J_{\mathrm{u}}^{*} \frac{\bar{c}_{\mathrm{M}}^{*}}{1+B n^{-1}}$. Finally, the derivative of the bioluminescence with respect to time at $t \rightarrow 0$ is written 


$$
\mathcal{L} \operatorname{um}(t)=k_{v} V_{\mathrm{T}} \frac{k_{\mathrm{f}} \tau_{\mathrm{q}}}{K_{\mathrm{Hi}}} S_{\mathrm{a}} c_{\mathrm{p}}^{\max } \frac{\bar{K}}{1+\bar{K}} J_{\mathrm{u}, 0}
$$

, which is eq 13 in the main text.

\section{SI-8. Analysis of the dependence of bioluminescence on time under conditions where non- linear metal biouptake and/or non-linear Hill regimes are operational (Figure S-3).}

In this section, eqs 4-6 and 8 are considered together with the full, non-linearized expressions of the metal biouptake flux (eqs 2-3) and of the Hill-based luciferase production rate. Figures S-3A,B illustrate how the Hill dissociation constant $\bar{K}_{\mathrm{Hi}}$ and the cooperativity index $n$ impact on the dimensionless bioluminescence $\bar{K}_{\mathrm{Hi}}^{-n} \mathcal{L} \mathrm{um}(\bar{t}) / \Omega$ (it is recalled that $\Omega \sim \bar{K}_{\mathrm{Hi}}^{-n}$ ). Increasing $\bar{K}_{\mathrm{Hi}}$ leads to a decrease in bioluminescence at short times (Figure S-3A), which is in line with the associated increase of the critical concentration in $\mathrm{MP}_{\text {reg }}$ complex required to 'switch on' half of the reporter genes. As a result, the maximum in bioluminescence is shifted to larger $\bar{t}_{\text {max }}$, which reflects the longer period necessary for filling the cells with the amount of $\mathrm{MP}_{\text {reg }}$ complex required to activate a significant fraction of reporter genes. These features explain in turn the existence of a crossover over time between the curves displayed in Figure S-3A for selected values of $\bar{K}_{\mathrm{Hi}}$. Qualitatively, similar trends are obtained when increasing $n$ (Figure S-3B) and they refer to the ease with which luciferase is produced upon modulation of the cooperativity of $\mathrm{MP}_{\text {reg }}$ binding to the promoter.

Figures S-3C and S-3D specify the dependence of $\mathcal{L u m}(\bar{t}) / \Omega$ on the concentration $\bar{c}_{\mathrm{M}, \mathrm{f}}^{*}$ of bioavailable $\mathrm{M}$ in solution and on the reciprocal bioavailability number $\mathrm{Bn}^{-1}$, respectively. An increase in $\bar{c}_{\mathrm{M}, \mathrm{f}}^{*}$ leads to increasing the biouptake flux $\bar{J}_{\mathrm{u}}(\bar{t})$ and, therefore, the bioluminescence, until metal transporter sites become saturated (i.e. $\bar{J}_{\mathrm{u}}(\bar{t}) \rightarrow 1$ ). This latter situation is achieved in the regime $\bar{c}_{\mathrm{M}, \mathrm{f}}^{*} \gg 1$ where bioluminescence then becomes independent of $\bar{c}_{\mathrm{M}, \mathrm{f}}^{*}$ (Figure S-3C). At short times, a significant limitation of the rate of metal biouptake by $\mathrm{M}$ diffusion from bulk medium to biosurface results in a decrease of $\bar{J}_{\mathrm{u}}$ and of $\mathcal{L} \mathrm{um}(\bar{t}) / \Omega$ (Figure S-3D). At sufficiently large $B n^{-1}$, i.e. when approaching the limit $x \rightarrow 0$, the maximum in bioluminescence disappears, which confirms the results detailed in SI-4 and Figure 2A. The existence of a crossover at sufficiently long $t$ between the curves computed for various $\mathrm{Bn}^{-1}$ further agrees with the trends displayed in Figure 2A, that is: light emission of the biosensors over longer delay with increasing $\mathrm{Bn}^{-1}$, and concomittant reduction of the maximum in 
bioluminescence, recalling that this maximum varies as $1 /\left(1+B n^{-1}\right)\left(\sim \bar{J}_{\mathrm{u}, 0}\right)$ in the linear Henry metal biouptake regime (Figure 2B). Figure S-3 evidences that the asymmetry of the bioluminescence peak is also determined by the nature of the process limiting the rate of metal bioputake (i.e. by the magnitude of $B n^{-1}$ ), by the Hill stability constant $\bar{K}_{\mathrm{Hi}}$ and the cooperativity index $n$ that both impact on the rate of luciferase production.
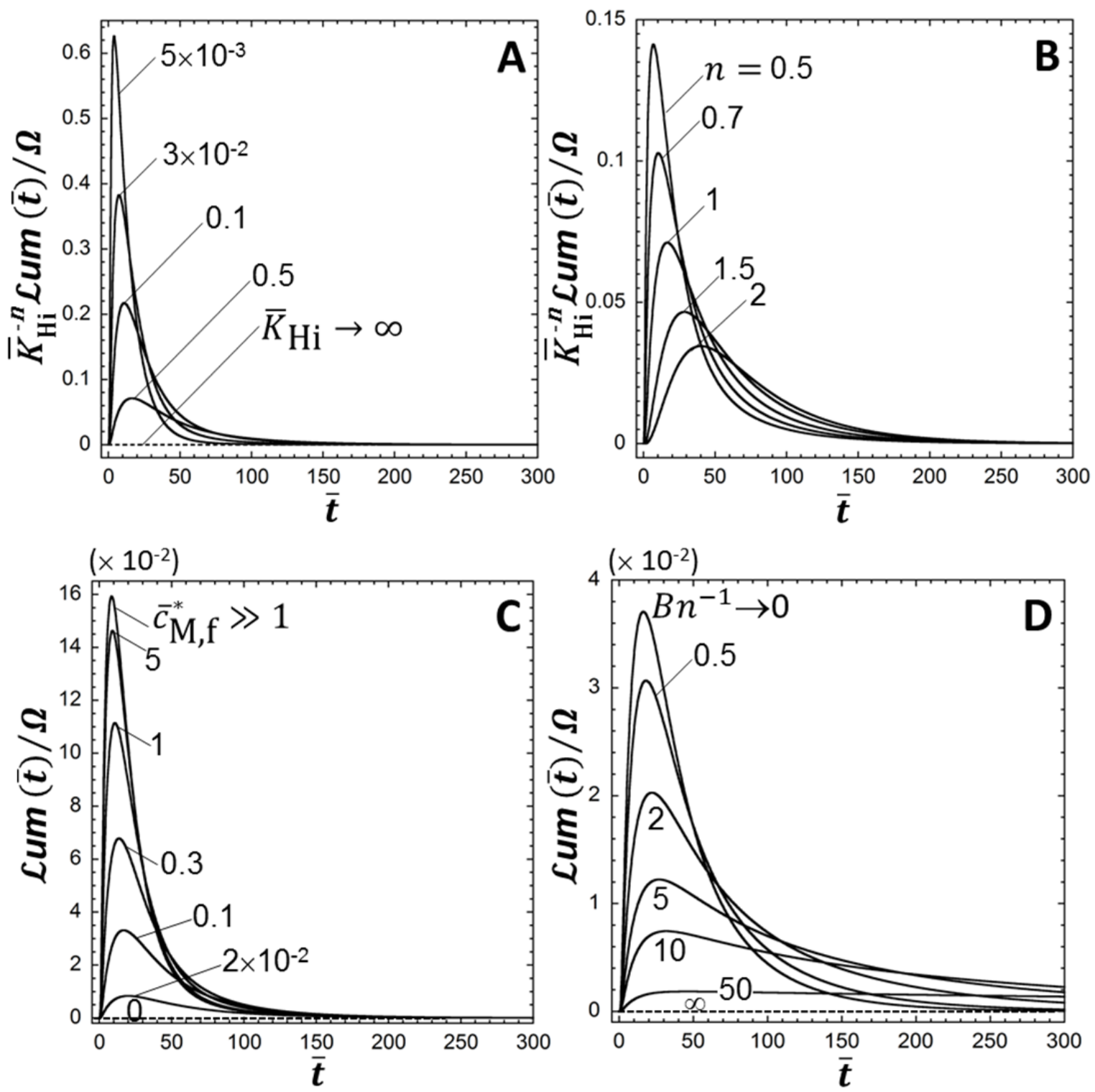

Figure S-3. Illustration of the impacts of $\bar{K}_{\mathrm{Hi}}(\mathbf{A}), n(\mathbf{B}), \overline{\mathrm{C}}_{\mathrm{M}, \mathrm{f}}^{*}(\mathbf{C})$ and $\mathrm{Bn}^{-1}(\mathbf{D})$ on the dependence of the dimensionless bioluminescence on time (eqs 2-3, 4-6, 8). Model parameters: $\tau_{\mathrm{q}} / \tau_{\mathrm{a}}=1, \bar{K}=10$, $1 /\left(k_{\mathrm{r}} \tau_{\mathrm{a}}\right)=10, k_{\mathrm{e}} \tau_{\mathrm{a}}=0.3, \varepsilon_{\mathrm{ads}}=0, \bar{c}_{\mathrm{p}}(t)=1$ and $(\mathbf{A}) n=1, B n^{-1}=0.1, \bar{c}_{\mathrm{M}, \mathrm{f}}^{*}=0.11 ;(\mathbf{B}) B n^{-1}=0.1$ , $\bar{c}_{\mathrm{M}, \mathrm{f}}^{*}=0.11 ; \bar{K}_{\mathrm{Hi}}=0.5 ;(\mathbf{C}) n=1, B n^{-1}=0.1, \bar{K}_{\mathrm{Hi}}=0.5 ;$ (D) $n=1, \bar{c}_{\mathrm{M}, \mathrm{f}}^{*}=0.11, \bar{K}_{\mathrm{Hi}}=0.5$. 


\section{SI-9. Gompertz expression for cell growth kinetics.}

The Gompertz expression for cell growth kinetics may be written in the form ${ }^{23,49,50}$

$$
\ln \left[\frac{c_{\mathrm{p}}(t)}{c_{\mathrm{p}}(0)}\right]=\rho \exp \left\{-\exp \left[\frac{\mathrm{e} \mu}{\rho}\left(T_{\mathrm{lag}}-t\right)+1\right]\right\} .
$$

, with $\mathrm{e}=\exp (1)$. In eq S64, $\mu\left(\mathrm{s}^{-1}\right)$ is the absolute (maximum) growth rate (defined by the tangent to the

curve $\ln \left(c_{\mathrm{p}}(t) / c_{\mathrm{p}}(0)\right)$ versus time taken at the inflexion point), $T_{\text {lag }}$ is the lag time required for the cells to significantly grow (e.g. as a result of their transfer from one medium to another differing in terms of nutrients bioavailability), and $\rho$ corresponds to $\ln \left[c_{\mathrm{p}}(t \rightarrow \infty) / c_{\mathrm{p}}(0)\right]$ and thus refers to the maximum population size the medium can sustain under given nutrient conditions. From eq S64, we infer that the dimensionless concentration of active metal-sensing cells $\bar{c}_{\mathrm{p}}(\bar{t})=c_{\mathrm{p}}(\bar{t}) / c_{\mathrm{p}}^{\max }$ can be formulated according to

$$
\bar{c}_{\mathrm{p}}(t)=\exp \left\{\rho \exp \left\{-\exp \left[\frac{\mathrm{e} \mu}{\rho}\left(T_{\mathrm{lag}}-t\right)+1\right]\right\}-\rho\right\}
$$

or, equivalently,

$$
\bar{c}_{\mathrm{p}}(\bar{t})=\exp \left\{\rho\left[\exp \left\{-\exp \left[\frac{\bar{T}_{\mathrm{lag}}-\bar{t}}{\bar{\alpha}}+1\right]\right\}-1\right]\right\}
$$

, where we introduced the dimensionless lag time $\bar{T}_{\text {lag }}=T_{\text {lag }} / \tau_{\mathrm{a}}$ and the dimensionless growth rate $1 / \bar{\alpha}$ defined by $\bar{\alpha}^{-1}=\frac{\mathrm{e} \mu}{\rho} \tau_{\mathrm{a}}$. Figures $\mathbf{3 A}$ and $\mathbf{3 B}$ in the main text correspond to evaluations of the bioluminescence for different values of $\bar{T}_{\text {lag }}$ (at fixed $\rho$ and $\bar{\alpha}$ ) and of $\bar{\alpha}$ (at fixed $\rho$ and $\bar{T}_{\text {lag }}$ ), respectively. Figure 4 displays simulations of $\mathcal{L} u m(\bar{t}) / \Omega$ for selected values of $\rho$ (at fixed $\bar{\alpha}$ and $\bar{T}_{\text {lag }}$ ). The case $\bar{c}_{\mathrm{p}}(\bar{t})=1$ in Figures 3-4 (curve (a) therein) simply corresponds to the formal limit $\rho=0$.

Examination of the experimental data displayed in Figure 5A reveals the absence of bioluminescence peak at very short time (i.e. at $t \rightarrow 0$ ). Following the results derived from Figure 4 (curve (g)) and discussed in the main text, this observation leads to the conclusion that the concentration of photoactive cells must satisfy the condition $\bar{c}_{\mathrm{p}}(t=0)=0$ under the experimental conditions of interest. To constrain 
the analysis of the experimental data on the basis of eqs 15 and 17, the required eq S65 is then forced to obey the condition $\bar{c}_{\mathrm{p}}(t=0)=0$ via the transformation

$$
\bar{c}_{\mathrm{p}}(t)=\frac{\exp \left\{\rho\left[\exp \left\{-\exp \left[\frac{T_{\mathrm{lag}}-t}{\alpha}+1\right]\right\}-1\right]\right\}-\exp \left\{\rho\left[\exp \left\{-\exp \left[\frac{T_{\mathrm{lag}}}{\alpha}+1\right]\right\}-1\right]\right\}}{1-\exp \left\{\rho\left[\exp \left\{-\exp \left[\frac{T_{\mathrm{lag}}}{\alpha}+1\right]\right\}-1\right]\right\}}
$$

, which satisfies $\bar{c}_{\mathrm{p}}(t=0)=0$ and $\bar{c}_{\mathrm{p}}(t \rightarrow \infty)=1$.

\section{SI-10. Demonstration of eq 17.}

Equation 16 may be rewritten in the form

$$
\begin{aligned}
\tilde{f}(t)= & \frac{\bar{K}}{\left(1-\frac{x}{k_{\mathrm{r}} \tau_{\mathrm{a}}}+\bar{K}\left(1+x-k_{\mathrm{r}} \tau_{\mathrm{a}}\right)\right)} \times \\
& \left\{\frac{(\Lambda \bar{K})^{-1}}{4}\left(1-\frac{2 x}{k_{\mathrm{r}} \tau_{\mathrm{a}}}+\bar{K}(1+x)\right)\left(\mathrm{e}^{-\frac{t}{\tau_{-}}}-\mathrm{e}^{-\frac{t}{\tau_{+}}}\right)-\left(\mathrm{e}^{\left.\left.-\frac{t}{\tau_{-}}+\mathrm{e}^{-\frac{t}{\tau_{+}}}\right)+\mathrm{e}^{-k_{\mathrm{r}} t}\right\}}\right.\right.
\end{aligned}
$$

, where we have introduced the time constants $\tau_{ \pm}$(s) defined by

$$
1 / \tau_{ \pm}=\delta_{ \pm}\left[\Lambda \pm \frac{1+\bar{K}(1+x)}{2 \bar{K}}\right] / \tau_{\mathrm{a}} .
$$

, with $\delta_{+}=1$ and $\delta_{-}=-1$. Recalling that $\Lambda$ is defined by eq S16, the Taylor expansions of eq S69 in the limit $\bar{K}>>1$ leads to $1 / \tau_{+} \approx(x+1) / \tau_{\mathrm{a}}$ and $1 / \tau_{-} \approx x /\left[\bar{K} \tau_{\mathrm{a}}(x+1)\right]$, which further simply into $1 / \tau_{+} \approx 1 / \tau_{\mathrm{a}}$ and $1 / \tau_{-} \approx x /\left(\bar{K} \tau_{\mathrm{a}}\right)$ for $x<<1$, respectively. Inspection of the rigorous dependence of $\tau_{ \pm} / \tau_{\mathrm{a}}$ on $\bar{K}$ (not shown) reveals that the aforementioned approximate expressions for $\tau_{ \pm}$satisfactorily apply for $\bar{K}$ that is a few times larger than unity. In addition, the quantity $\frac{(\Lambda \bar{K})^{-1}}{4}\left(1-\frac{2 x}{k_{\mathrm{r}} \tau_{\mathrm{a}}}+\bar{K}(1+x)\right)$ involved in eq S68 reduces to $\frac{1}{2}-\frac{x}{k_{\mathrm{r}} \tau_{\mathrm{a}} \bar{K}}$ in the limits $\bar{K}>>1, x<<1$ and $k_{\mathrm{r}} \tau_{\mathrm{a}}<<1$. Finally, the prefactor $\frac{\bar{K}}{\left(1-\frac{x}{k_{\mathrm{r}} \tau_{\mathrm{a}}}+\bar{K}\left(1+x-k_{\mathrm{r}} \tau_{\mathrm{a}}\right)\right)}$ in eq $\mathrm{S} 68$ can be approximated by $\left(1-\frac{x}{k_{\mathrm{r}} \tau_{\mathrm{a}} \bar{K}}\right)^{-1}$ under the here 
examined limiting conditions. In turn, eq S68 reduces to eq 17 given in the main text after using the inequality $\tau_{+} / \tau_{-} \approx x / \bar{K}<<1$ and introducing $k_{\mathrm{eff}}=x /\left(\bar{K} \tau_{\mathrm{a}}\right)$. 